\title{
On a Linear Structure of the Quotient Variety by a Finite Reflexion Group ${ }^{\dagger)}$
}

Dedicated to Professor Heisuke Hironaka on the occasion of his 60 th birthday

By

Kуoji Sarto*

The objective of the article is to show that the orbit space of a finite reflection group acting on the complexification of the real vector space carries naturally a complex vector space structure $\Omega$ together with a nondegenerate bilinear form $J$ on it defined over the real number field. For details of the results, one is referred to Theorems I, II and III in the introduction. This structure on the invariants is called the flat structure.

Originaly, the flat structure (published later in [7], Oda [24]) is defined on the deformation space of an isolated hypersurface singularity via Gauss-Manin connection and higher residue theory in general. On the other hand, the deformation of a simple singularity is described in terms of a simple Lie algebra. Namely, the deformation space is given by the quotient space of the Cartan algebra by the action of the Weyl group (Brieskorn [2], Slodowy [33]). This suggests that the flat structure is described in terms only of the Weyl group in that case. Actually, such a description is achieved in the present article so that the above mentioned flat structure on the quotient space of a finite reflection group is obtained. The key fact in the construction of the flat metric is the regularity of the eigenvector for exp $(2 \pi \sqrt{-1} / h)$ of a Coxeter element with the Coxeter $\#=h$ (cf. [6], [15], [34]).

Since the present article was written (cf. the Footnote $\left.{ }^{*}\right)$ ), there have been several developments in the study of the flat structure and its generalization, as will be summarized below. Nevertheless the present article

†) Except for the new additional introduction and the updated references [13]-[40], this article, up to minor changes is exactly the same as the preprint RIMS-288, Kyoto University, June 1979.

Received May 7, 1991.

1991 Mathematics Subject Classifications: 14H30, 14H60, 30F10, 30F35.

* RIMS, Kyoto University, Sakyo-ku, Kyoto, 606-01, Japan. 
gives a systematic definition and a complete proof of the existence of the flat structure for all finite reflection groups (including Weyl groups of simple Lie algebras) which has not yet been covered by other articles. On the other hand, the subject gets a new impetus from conformal field theory in physics. Thus it seems to be of interest to publish this in the original form without any changes, even though it missed a conceptual explanation at that time.

The rest of this new introduction is devoted to give a brief review on some subsequent developments. For more results on related subjects, the readers are referred to the bibliographs in the papers quoted below. We shall distinguish between the flat structures defined on the invariants of reflection groups and those defined on the deformation spaces by calling them flat invariants and flat coordinates respectively.

\section{Definition of flat structure.}

i) The definition of the flat structure on the deformation space of an isolated singularity is given in [7], [24] (cf. [21], [27], [28] for the higher residue theory).

ii) For a general reflection group, there is no definition of flat invariants, for which a notion of a Coxeter element is necessary. At this moment, the notion of flat invariants is established for finite reflection group in [9] and the present paper, and for extended affine Weyl groups in [29].

\section{Simple singularity and finite reflection group.}

i) The flat coordinates for simple singularities from the view point of the Gauss-Manin connection are carried out by several authors:

a) The $A_{\ell}$-type singularities can be treated simultaneously by a use of generating function [18]. For the singularities of type $E_{6}, E_{7}$ and $E_{8}$, it is done in [37], [38] and [19].

b) A systematic determination of the flat coordinates for all simple singularities by a use of $\delta_{1}$-expansion is carried out in [22].

c) Another concrete and detailed description of their Gauss-Manin system for the types $A_{\ell}$ and $D_{\ell}$ is given in [13].

d) The subspace, corresponding to a folding of a Dynkin graph, in the deformation space of a simple singularity is linear with respect to the flat structure [40].

e) The theory of Brieskorn-Slodowy is reconstructed by using geometry 
of momentum maps, which leads to a construction of a primitive form [36].

ii) The explicit case by case determination of the flat invariants for all finite reflection groups except for the types $E_{7}$ and $E_{8}$ is done in [9]. The flat invariants for the group $E_{7}$ are given in [39].

\section{Simple elliptic singularity and extended affine root system.}

i) Simple elliptic singularities are introduced in [26] and their primitive forms are given in [7]. Tables of the flat coordinates for simple elliptic singularities are in [20], [23].

ii) For a root system belonging to a positive semidefinite Killing form of 2-dimensional radical, called an extended affine root system, one has a Coxeter element with regular eigenvectors, which makes it possible to construct flat metric on the invariants and to introduce the flat invariants [29]. The modular property of the flat invariants is studied in [31] and the flat invariants are being described by the Jacobi forms [32] (cf. [35]). A survey is given in [30].

\section{Unitary reflection group.}

The concept of the flat structure can be generalized to a certain class of unitary reflection groups [25].

\section{Deformation of conformal field theory.}

In the topological conformal field theory, one finds the same mathematical structure as the theory of flat coordinates and higher residue pairings ([16], [17] and [14]).

\section{Introduction}

Let $V$ be a real $\ell$-dimensional vector space and $W$ be a finite group of linear transformations of $V$ generated by reflexions. Suppose that the action of $W$ on $V$ is irreducible. The group $W$ acts also on the symmetric $\boldsymbol{R}$-algebra $S$ of $V$. Let us denote by $R=S^{W}$ the subalgebra of $S$ of all invariants of $S$. Then $R$ is generated by $\ell$ algebraically independent homogeneous elements whose degrees $m_{1}+1, \cdots, m_{\ell}+1$ are described by the eigenvalues of a Coxeter transformation. This fact was shown by Coxeter using the classification of finite reflexion groups. Then Chevalley, Coleman and Steinberg gave other proofs without using the classification (see Bourbaki 
[1] Chap. 5).

Let us denote by $\operatorname{Der}_{R}$ the $R$-module of all $R$-derivations of $R$. In this note in $\S 5(5.4)$, we introduce canonically a non-degenerate $R$-bilinear form,

$$
\tilde{J}: \operatorname{Der}_{R} \times \operatorname{Der}_{R} \rightarrow R \text {. }
$$

Then the main purpose of this note is to show the following theorems (see (10.5), (10.6) and (10.7)).

Theorem I. There exist an $\ell$-dimensional $R$-vector subspace $\Omega \subset \operatorname{Der}_{R}$ such that

1) $\Omega$ has a direct sum decomposition $\Omega=\oplus \Omega^{\left(m_{i}+1\right)}$ such that any element of $\Omega^{\left(m_{i}+1\right)}$ is homogeneous of degree-( $\left.m_{i}+1\right)$.

2) $\Omega$ generates $\operatorname{Der}_{R}$ : i.e. $\operatorname{Der}_{R} \simeq \Omega \otimes_{R} R$.

3) $\Omega$ is integrable: i.e. $\left[\delta_{1}, \delta_{2}\right]=0$ for any $\delta_{1}, \delta_{2} \in \Omega$.

4) The restriction of $\tilde{J}$ on $\Omega \times \Omega$ takes constant values in $\mathbb{R}$. Hence we get a non-degenerate $\mathbb{R}$-bilinear form,

$$
J: \Omega \times \Omega \rightarrow R .
$$

Theorem II. Conversely to the theorem $I$, if a vector subspace $\Omega^{\prime} \subset \operatorname{Der}_{R}$ satisfies conditions 2$\left.)^{\prime}, 3\right)^{\prime}$ and 4$)^{\prime}$ (replace $\Omega$ by $\Omega^{\prime}$ in the conditions 2$), 3$ ) and 4) of the theorem I), then $\Omega^{\prime}=\Omega$.

Theorem III. Put $\Omega^{*}:=\left\{P \in R^{+} ; \delta P \in R\right.$ for $\left.\forall \delta \in \Omega\right\}$.

Then, 1) $\Omega^{*}$ is naturally a dual vector space of $\Omega$.

2) The algebra $R$ is generated by $\Omega^{*}$.

Corollary. Spec $R$ has a structure of a vector space $\Omega$ with an inner product $J$.

In a previous paper [9], we studied such vector space $\Omega^{*} \subset R$ of the above theorem. We called an $R$-basis of $\Omega^{*}$, a flat generator system of $R$ and have shown the existence of a flat generator system for each type (except $E_{7}$ and $E_{8}$ ) of groups separately. (See Theorem (1.14) and Definition (1.15) of the note.) In this note, we give a systematic (including $E_{7}$ and $\left.E_{8}\right)$ proof of existence of $\Omega$ by the use of a connection with logarithmic pole [8]

Originally this note was planned as a part of the theory of primitive integrals [7], for the application to the study of universal unfolding of simple 
singularities (see Brieskorn [2]), from where the problem of this note has started. Since the things treated in this note can be read relatively independently of the whole theory, we publish this part separately. Nevertheless the whole idea of constructions are due to the theory of primitive integrals, such as the use of a primitive vector field $D^{(h)}$ and the reduction of the coefficient ring from $R$ to $T:=\operatorname{ker}\left(D^{(h)}\right)$ and the reductions of $\operatorname{Der}_{R}$ to $\mathscr{G}$ and $\Omega_{R}$ to $\mathscr{F}$ in $\S 2$, computation of the multiplicity of the discriminant $\Delta^{2}=\operatorname{dim} V$ in $\S 3$, use of $\operatorname{Der}_{R}\left(\log \Delta^{2}\right), \Omega_{R}\left(\log \Delta^{2}\right)$ and several dualities between them by the use of $\tilde{I}$ and $\tilde{I}^{*}$ in $\S 4$, use of $v$ and $w$ and the comparison of $\tilde{I}$ and $\tilde{J}$ by $\tilde{J}^{*}=D^{(h)} \tilde{I}^{*}$ and $\tilde{J}=\tilde{I}(w(\cdot), \cdot)$ in $\S 5$ and $\S 6$, the use of Euler operator $E$ in $\S 7$, study of a torsion free connection $\nabla$ with $\nabla \tilde{I}^{*}=0$ in $\S 8$, study of a torsion free connection $\nabla /$ with $\nabla / \tilde{J}^{*}=0$ and the integrability of $\nabla /$ in $\S 9$ and the introduction of a linear space $\Omega:=\operatorname{ker} \nabla /$ in $\S 10$.

It must be noted that our construction of $\Omega, \Delta^{2}, J$ from $W, V, I$ is just the reversed direction of the construction of Poincare duality of vanishing cycles of simple singularities from the duality of certain local cohomology groups (for detail see $\S 11$ and [7]).

\section{Contents}

$\S 1$. Basic notations and results on finite reflexion groups

$\S 2$. $D^{(h)}$ and the reduction of $R$ to $T, \operatorname{Der}_{R}$ to $\mathscr{G}$ and $\Omega_{R}$ to $\mathscr{F}$

$\S 3$. The multiplicity of $\Delta^{2}$ of $R$

$\S 4$. $\operatorname{Der}_{R}\left(\log \Delta^{2}\right), \Omega_{R}\left(\log \Delta^{2}\right)$ and dualities by $\tilde{I}$ and $\tilde{I}^{*}$

$\S 5$. Bilinear form $\tilde{J}: \operatorname{Der}_{R} \times \operatorname{Der}_{R} \rightarrow R$

$\S 6$. Graduation of $\operatorname{Der}_{R}$ and $\Omega_{R}$ by the order of $P_{\ell}, v$ and $w$

$\S 7$. The Euler operator $E$

$\S 8$. Torsion free connection $\nabla$ with $\nabla \tilde{I}^{*}=0$

$\S 9$. Torsion free connection $\nabla /$ with $\nabla / \tilde{J}^{*}=0$

$\S 10$. Space $\Omega=\oplus \Omega^{\left(m_{i}+1\right)}$

$\S 11$. Concluding remarks

\section{$\S 1$. Basic Notations and Results on Finite Reflexion Groups}

In this paragraph we repeat basic notations and results on the invariant rings of finite reflexion groups from N. Bourbaki [1] Chap. 5.

i) Let $V$ be a real $\ell$-dimensional vector space and let $W$ be a finite subgroup of $G L(V)$ generated by reflexions. Then the isomorphism type of $W$ is given by one of the following symbols: $A_{\ell}, B_{\ell}, D_{\ell}, E_{6}, E_{7}, E_{8}, F_{4}, G_{2}, H_{3}$, 
$H_{4}, I_{2}(p)$.

ii) Let $S$ be the symmetric algebra of $V$ with the natural graded ring structure, $S=\bigoplus_{d=0}^{\infty} S^{(d)}$. (i.e. $S$ is an $R$-algebra generated by $V$.) An element $P$ of $S^{(d)}$ is called homogeneous of degree $d$.

iii) The action of $W$ on $V$ is naturally extended to the action on the algebra $S$. We denote by $R$ the subring $S^{W}$ of $S$ of all $W$-invariant elements of $S$. $\quad R$ inherits a graded ring structure $R=\bigoplus_{d=0}^{\infty} R^{(d)}$ from $S$, by $R^{(d)}:=R \cap S^{(d)}$, $d=0,1,2, \cdots . \quad$ Put $R^{+}=\underset{d>0}{\bigoplus} R^{(d)}$.

iv) As an $R$-module, $S$ is $R$-free of rank $\# W$.

v) As an $R$-algebra, $R$ is generated by $\ell$ homogeneous elements $P_{1}, \cdots, P_{\ell} \in S . \quad P_{1}, \cdots, P_{\ell}$ are algebraically independent over $\boldsymbol{R}$. Thus the invariant ring $R$ is isomorphic to a polynomial algebra $\mathbb{R}\left[P_{1}, \cdots, P_{\ell}\right]$.

Note that this description of $R$ is not canonical, since the generator system $P_{1}, \cdots, P_{\ell}$ is not canonically determined.

vi) Let $\Sigma$ be the set of reflexions in $W$ which is not equal to 1 .

Then there is an equality

$$
\sum_{i=1}^{\ell}\left(\operatorname{deg} . P_{i}-1\right)=\# \Sigma \text {. }
$$

vii) A reflexion $g \in \Sigma$ is described by $g(x)=x+f_{g}(x) e_{g} x \in V$, for some $e_{g} \in V$ and $f_{g} \in V^{*}$. Put $\Delta:=\prod_{g \in \Sigma} e_{g} \in S$.

viii) An element $P \in S$ is called anti-invariant, if $g(P)=(\operatorname{det} g)^{-1} P$ for $g \in W$. Then

a) The set of all anti-invariants of $W$ coincides with $\Delta R$.

b) By a choice of a linear basis $X_{1}, \cdots, X_{\ell}$ of $V$, we identify $S$ with a polynomial ring $R\left[X_{1}, \cdots, X_{\ell}\right]$. Let $P_{1}, \cdots, P_{\ell}$ be a system of homogeneous generators of the algebra $R$. Then the Jacobian det. $\left[\frac{\partial\left(P_{1}, \cdots, P_{\ell}\right)}{\partial\left(X_{1}, \cdots, X_{\ell}\right)}\right]$ is of the form $\lambda \Delta$ for some constant $\lambda \in R^{*}$.

ix) Let $c \in W$ be a Coxeter transformation whose eigenvalues are $\exp \left(2 \pi \sqrt{-1} m_{i} / h\right), i=1, \cdots \ell$, where $h$ is the Coxeter number of $W$ and $m_{1}=1<m_{2} \leq \cdots \leq m_{\ell-1}<m_{\ell}=h-1$ and $m_{i}+m_{\ell+1-i}=h$ for $i=1, \cdots, \ell$.

Then by a suitable change of the order,

$$
\text { deg. } P_{i}=m_{i}+1, \quad i=1, \cdots, \ell \text {. }
$$


Hence, $\# \Sigma=\sum_{i=1}^{\ell} m_{i}=(1 / 2) h \ell$.

x) Let $I \in R$ be a $W$-invariant form of degree 2 , which is unique up to a constant factor. By a suitable choice of a sign, $I$ is a positive definite symmetric bilinear form

$$
I: V^{*} \times V^{*} \rightarrow \boldsymbol{R}
$$

where $V^{*}$ is the $R$-dual space of $V$. Thus $I$ induces an isomorphism $V^{*} \simeq V$. By this identification, one gets a $W$-invariant symmetric positive definite bilinear form $I^{*}: V \times V \rightarrow \boldsymbol{R}$.

\section{$\S 2 . D^{(h)}$ and the Reduction of $R$ to $T, \operatorname{Der}_{R}$ to $\mathscr{G}$ and $\Omega_{R}$ to $\mathscr{F}$}

(2.1) Definition. We define following graded $S$ or $R$ modules.

i) $\operatorname{Der}_{s}:=\left\{\delta \in \operatorname{Hom}_{R}(S, S): \delta(P Q)=(\delta P) Q+P(\delta Q)\right.$ for all $\left.P, Q \in S\right\}$

$\Omega_{S}:=\operatorname{Hom}_{S}\left(\operatorname{Der}_{S}, S\right)$.

Then $\operatorname{Der}_{S}$ and $\Omega_{S}$ are free $S$-modules of rank $\ell$ with basis $\frac{\partial}{\partial X_{1}}, \cdots, \frac{\partial}{\partial X_{\ell}}$ and dual basis $d X_{1}, \cdots, d X_{\ell}$.

An element $\delta \in \operatorname{Der}_{S}$ is homogeneous of degree $d$, if $\delta S^{(k)} \subset S^{(k+d)}$, $k=0,1,2, \cdots$. Hence we get graded $S$-module structures, $\operatorname{Der}_{s}=\bigoplus_{d=-1}^{\infty}$ $\left(\operatorname{Der}_{S}\right)^{(d)}$ and $\Omega_{S}=\bigoplus_{d=1}^{\infty}\left(\Omega_{S}\right)^{(d)}$.

ii) $\operatorname{Der}_{R}:=\left\{\delta \in \operatorname{Hom}_{R}(R, R): \delta(P Q)=(\delta P) Q+P(\delta Q)\right.$ for $\left.P, Q \in R\right\}$

$\Omega_{R}:=\operatorname{Hom}_{R}\left(\operatorname{Der}_{R}, R\right)$.

Then $\operatorname{Der}_{R}$ and $\Omega_{R}$ are free $R$-modules of rank $\ell$ with basis $\frac{\partial}{\partial P_{1}}, \cdots, \frac{\partial}{\partial P_{\ell}}$ and dual basis $d P_{1}, \cdots, d P_{\ell}$.

An element $\delta \in \operatorname{Der}_{R}$ is homogeneous of degree $d$, if $\delta R^{(k)} \subset R^{(k+d)}$, $k=0,2, \cdots$. Hence we get graded $R$-module structures $\operatorname{Der}_{R}=\bigoplus_{d=-h}^{\infty}\left(\operatorname{Der}_{R}\right)^{(d)}$ and $\Omega_{R}=\bigoplus_{d=2}^{\infty}\left(\Omega_{R}\right)^{(d)}$

(2.2) Let us choose and fix a generator $D^{(h)}$ of the one dimensional vector space $\left(\operatorname{Der}_{R}\right)^{(-h)}($ cf. $\left.\S 1 \mathrm{ix})\right)$, which is spanned by $\frac{\partial}{\partial P_{\ell}}$. 


\section{Definition.}

$T:=\left\{P \in R: D^{(h)} P=0\right\}$

$\mathscr{F}:=\left\{\omega \in \Omega_{R}: L_{D^{(h)}} \omega=0\right.$, where $L_{D^{(h)}}$ is the Lie derivative of $\left.L^{(h)}\right\}$

$\mathscr{G}:=\left\{\delta \in \operatorname{Der}_{R}:\left[D^{(h)}, \delta\right]=0\right.$, where [, ] is the bracket product $\}$

Here, one recalls that the $\mathrm{Lie}$ derivative and the bracket product are related by the relation: $<L_{\delta} \omega, \delta^{\prime}>=\delta<\omega, \delta^{\prime}>-<\omega,\left[\delta, \delta^{\prime}\right]>$ for any $\delta, \delta^{\prime} \in \operatorname{Der}_{R}$ and $\omega \in \Omega_{R}$ with respect to the natural pairing $<*, *>$ between $\operatorname{Der}_{R}$ and $\Omega_{R}$.

(2.3) One may check easily the following assertions.

i) $T$ is a polynomial $R$-algebra generated by homogeneous elements, $P_{1}, \cdots, P_{\ell-1}$ of (1) v). Hence $R$ is a polynomial ring $T\left[P_{\ell}\right]$ with one indeterminate over $T$.

ii) $\mathscr{F}$ is a graded free $T$-module of rank $\ell$, such that we have an isomorphism $\mathscr{F} \otimes_{T} R \simeq \Omega_{R}$.

We have an inclusion $d \mathscr{F} \subset \mathscr{F} \wedge \mathscr{F} . \quad(\because$ As a $T$-module, $\mathscr{F}$ is generated by $d P_{1}, \cdots, d P_{\ell}$.)

iii) $\mathscr{G}$ is a graded free $T$-module of rank $\ell$, such that we have an isomorphism $\mathscr{G} \otimes \otimes_{T} R \simeq \operatorname{Der}_{R}$.

The module $\mathscr{G}$ is closed under the bracket product. $(\because$ As a $T$-module, $\mathscr{G}$ is generated by $\frac{\partial}{\partial P_{1}}, \cdots, \frac{\partial}{\partial P_{\ell}}$.)

iv) The canonical pairing $\operatorname{Der}_{R} \times \Omega_{R} \rightarrow R$ induces a $T$-bilinear form $\mathscr{F} \times \mathscr{G} \rightarrow T$, so that each of $\mathscr{F}$ and $\mathscr{G}$ is a dual $T$-module of the other.

(2.4) Let us introduce an increasing sequence $\left\{\mathscr{F}^{\left(m_{i}+1\right)}\right\}_{i=1, \cdots, \ell}$ of $T$ submodules of $\mathscr{F}$, and a decreasing sequence $\left\{\mathscr{G}^{\left(m_{i}+1\right)}\right\}_{i=1, \cdots, \ell}$ of $T$-submodules of $\mathscr{G}$ as follows.

\section{Definition.}

i) $\mathscr{F}^{\left(m_{i}+1\right)}:=\sum_{\substack{P \in R \\ \operatorname{deg} P \leq m_{i}+1}} T d P, i=1, \cdots, \ell$.

ii) $\mathscr{G}^{\left(m_{i}+1\right)}:=\left\{\delta \in \mathscr{G}: \delta \cdot \omega=0\right.$ for $\left.{ }^{\forall} \omega \in \mathscr{F}^{(k)}, k \leq m_{i}\right\}, i=1, \cdots, \ell$. 
(2.5) Let $P_{1}, \cdots, P_{\ell}$ be a system of homogeneous generators of the algebra $R$ of (1) v). Then we have presentations,

$$
\begin{aligned}
& \mathscr{F}^{(d)}=\underset{m_{\imath}+1 \leq d}{\bigoplus} T d P_{i} \\
& \mathscr{G}^{(d)}=\bigoplus_{m_{i}+1 \geq d} T \frac{\partial}{\partial P_{i}},
\end{aligned}
$$

so that we have the following assertion.

i) $\quad 0 \varsubsetneqq \mathscr{F}^{(2)} \varsubsetneqq \cdots \varsubsetneqq \mathscr{F}^{\left(m_{\mathrm{t}}+1\right)} \varsubsetneqq \cdots \varsubsetneqq \mathscr{F}^{(h)}=\mathscr{F}$

is an increasing sequence of graded free $T$-submodules of $\mathscr{F}$ such that $\mathscr{F}^{\left(m_{i}+1\right)} / \mathscr{F}^{\left(m_{j}+1\right)}$ is a $T$-free module of rank equal to $\sharp\left\{1 \leq k \leq \ell: m_{j}<m_{k} \leq m_{i}\right\}$

ii) $\mathscr{G}=\mathscr{G}^{(2)} \supsetneqq \cdots \supsetneqq \mathscr{G}^{\left(m_{i}+1\right)} \supsetneqq \cdots \supsetneqq \mathscr{G}^{(h)} \supsetneqq 0$

is a decreasing sequence of graded free $T$-submodules of $\mathscr{G}$ such that $\mathscr{G}^{\left(m_{j}+1\right)} / \mathscr{G}^{\left(m_{1}+1\right)}$ is a $T$-free module of rank equal to $\sharp\left\{1 \leq k \leq \ell: m_{j} \leq m_{k}<m_{i}\right\}$.

(2.6) i) The pairing $\mathscr{F} \times \mathscr{G} \rightarrow T$ induces zero homomorphisms,

$$
\mathscr{F}^{\left(d_{1}\right)} \times \mathscr{G}^{\left(d_{2}\right)} \rightarrow 0 \text { for } d_{1}<d_{2}
$$

ii) For $d \in\left\{m_{1}+1, \cdots, m_{\ell}+1\right\}$, let us denote by $d^{+}$the smallest element of $\left\{m_{1}+1 \cdots, m_{\ell}+1, \infty\right\} \cap\left\{d^{\prime}: d^{\prime}>d\right\}$ and denote by $d^{-}$the largest element of $\left\{0, m_{1}+1, \cdots, m_{\ell}+1\right\} \cap\left\{d^{\prime}: d^{\prime}<d\right\}$.

Then $\left(\mathscr{F}^{(d)}\right)^{\perp}=\mathscr{G}^{\left(d^{+}\right)}$and $\left(\mathscr{G}^{(d)}\right)^{\perp}=\mathscr{F}^{\left(d^{-}\right)}$

iii) As T-modules we have the following identifications,

$$
\left(\mathscr{F}^{(d)}\right)^{*} \cong \mathscr{G} / \mathscr{G}^{\left(d^{+}\right)} \text {and }\left(\mathscr{G}^{(d)}\right)^{*} \cong \mathscr{F} / \mathscr{F}^{\left(d^{-}\right)}
$$

The proofs of i), ii), and iii) are almost evident by the presentation of (2.5).

(2.7) Note. From the presentation of (2.5), Gr. $\left(\mathscr{F}^{(*)}\right)=\underset{d}{\bigoplus^{(d)}} / \mathscr{F}^{\left(d^{-)}\right.}$and Gr. $\left(\mathscr{G}^{(*)}\right):=\bigoplus_{d} \mathscr{G}^{(d)} / \mathscr{G}^{\left(d^{+}\right)}$are $T$-free modules of rank $\ell$. Later in (10.3), we shall give canonical identifications,

$$
\mathscr{F} \simeq \operatorname{Gr} .\left(\mathscr{F}^{(*)}\right) \quad \text { and } \quad \mathscr{G} \simeq \operatorname{Gr} .\left(\mathscr{G}^{(*)}\right)
$$




\section{$\S 3$. The Multiplicity of $\Delta^{2}$ in $R$}

The main purpose of this paragraph is to show that $\Delta^{2}$ is a monic polynomial of degree $\ell$ in $P_{\ell}$ over $T$ (cf. Lemma (3.1)).

(3.1) $\Delta S \cap R=\Delta^{2} R$ ( $\because$ If $\triangle P \in R$ for a $P \in S$, then $P$ is an anti-invariant and $P \in \Delta R$ by (1) viii) a).)

Let us develop $\Delta^{2} \in R=T\left[P_{\ell}\right]$ as a polynomial in $P_{\ell}$. Then the following lemma is crucial for all what will follow.

Lemma. $\Delta^{2}$ has the following development.

$$
\Delta^{2}=c P_{\ell}^{\ell}+A_{1} P_{\ell}^{\ell-1}+\cdots A_{\ell}
$$

where $c$ is a non-zero constant and $A_{i} \in T, i=1, \cdots, \ell$.

Proof. Since deg. $\Delta^{2}=2 \# \Sigma=h \ell=\ell \operatorname{deg} . P_{\ell}(\because(1)$ ix $\left.)\right)$, we have the form,

$$
\Delta^{2}=A_{0} P_{\ell}^{\ell}+A_{1} P_{\ell}^{\ell-1}+A_{2} P_{\ell}^{\ell-2}+\cdots+A_{\ell},
$$

where $A_{i} \in T$ and $\operatorname{deg} . A_{i}=h i, i=0,1, \cdots, \ell$.

Thus all we have to show is $A_{0} \neq 0$. It is enough to show that $\Delta^{2} \notin T^{+} R$ where $T^{+}:=T \cap R^{+}$. Let us show the following stronger assertion.

(3.2) Lemma. Consider the ideal $T^{+} S$ of $S$ generated by $T^{+}$. Then, $\Delta \notin$ radical of $T^{+} S$.

Proof. Let $\xi \in V^{*} \otimes_{R} C$ be an eigenvector of a transformation $c \in W$, such that $c \xi=\lambda \xi$, where $\lambda=\exp (2 \pi \sqrt{-1} q / p), p, q \in Z,(p, q)=1$. Let $P \in R$ be a homogeneous invariant polynomial of degree $d$.

Then, $P(\xi)=P(c \xi)=P(\lambda \xi)=\lambda^{d} P(\xi)$.

Hence if $p \nmid d$, then $P(\xi)=0$.

Now let $c$ be a Coxeter transformation and $\xi \neq 0$ be an eigenvector belonging to the eigenvalue $\exp (2 \pi \sqrt{-1} / h)$. Since $1<\operatorname{deg} . P_{j}<h$ for $j=1, \cdots, \ell-1(\because(1) \mathrm{ix})$ ), we conclude $P_{1}(\xi)=\cdots=P_{\ell-1}(\xi)=0$. Since the ideal $T^{+} S$ is generated by $P_{1}, \cdots, P_{\ell-1}$, the vector $\xi \in V^{*} \otimes_{R} C$ is a root of the ideal $T^{+} S$.

On the other hand, $\xi$ is not contained in any hyperplane of a reflexion of $W\left(\because[1]\right.$ chap. $\mathrm{V}, \S 6, \mathrm{n}^{\circ} 2$, note). This means $\Delta(\xi) \neq 0$. Hence $\Delta \notin$ 
radical of $T^{+} S$.

q.e.d.

(3.3) Note 1. Since $R=T\left[P_{\ell}\right]$, the Lemma (3.1) is equivalent to the following statement.

Assertion. $R / \Delta^{2} R$ is a $T$-free module of rank $\ell$. In particular $R / \Delta^{2} R$ is integral over $T$.

Note 2. Let $e^{1}, \cdots, e^{\ell} \in R / \Delta^{2} R$ be a $T$-free basis. Let us present by a matrix $A$ the endomorphism of $R / \Delta^{2} R$ defined by multiplication by $P_{\ell}$.

$$
P_{\ell} e^{i}=\sum_{j=1}^{\ell} A_{j}^{i} e^{j}, A_{j}^{i} \in T, i, j=1, \cdots, \ell .
$$

Then one may construct an $R$-free resolution of $R / \Delta^{2} R$,

$$
0 \rightarrow(R)^{\ell} \stackrel{M}{\rightarrow}(R)^{\ell} \stackrel{N}{\rightarrow} R / \Delta^{2} R \rightarrow 0
$$

where $N\left(Q_{1}, \cdots, Q_{\ell}\right)=\sum_{i=1}^{\ell} Q_{i} e^{i}$

$$
M\left(R_{1}, \cdots, R_{\ell}\right)=\left(\sum_{i=1}^{\ell}\left(P_{\ell} \delta_{j}^{i}-A_{j}^{i}\right) R_{i}\right)_{j=1, \cdots, \ell}
$$

Then we obtain a presentation of $\Delta^{2}$ by the determinant of $M$.

$\operatorname{det} . M=\operatorname{det}\left(P_{\ell} I_{\ell}-A\right)=c \Delta^{2}$ for a non-zero constant $c \in R^{*}$.

\section{$\S 4 . \operatorname{Der}_{R}\left(\log \Delta^{2}\right), \Omega_{R}\left(\log \Delta^{2}\right)$ and Dualities by $\tilde{I}$ and $\tilde{I}^{*}$}

In this paragraph, we introduce $\operatorname{Der}_{R}\left(\log \Delta^{2}\right)$ and $\Omega_{R}\left(\log \Delta^{2}\right)$ and then give a description of them by a use of $\tilde{I}$ and $\tilde{I}^{*}$ (cf. Theorem (4.6)).

(4.1) Since $V^{*}$ can be identified with its tangent space, and $V$ can be identified with the cotangent space of $V^{*}$ at anypoint of $V^{*}$, we may extend the bilinear forms $I$ and $I^{*}$ on $V^{*}$ and $V$ of (1) x), to the tangent bundle and cotangent bundle of $V^{*}$. We shall denote the extended bilinear forms by $\tilde{I}$ and $\tilde{I}^{*}$.

Thus we get symmetric $S$-bilinear forms,

$\tilde{I}: \operatorname{Der}_{s} \times \operatorname{Der}_{s} \rightarrow S, \tilde{I}\left(\frac{\partial}{\partial X_{i}}, \frac{\partial}{\partial X_{j}}\right):=\frac{\partial^{2}}{\partial X_{i} \partial X_{j}} I$ for $i, j=1, \cdots, \ell$ 
$\tilde{I}^{*}: \Omega_{S} \times \Omega_{S} \rightarrow S, \tilde{I}^{*}\left(d X_{i}, d X_{j}\right):=I\left(X_{i}, X_{j}\right)$ for $i, j=1, \cdots, \ell$.

Since $\tilde{I}$ and $\tilde{I}^{*}$ are non-degenerate, we get $S$-isomorphisms

$$
\begin{aligned}
& \tilde{I}: \operatorname{Der}_{S} \rightarrow\left(\operatorname{Der}_{S}\right)^{*} \simeq \Omega_{S}, \tilde{I}(\delta):=\tilde{I}(\delta, \cdot) \text { for } \delta \in \operatorname{Der}_{s} \\
& \tilde{I}^{*}: \Omega_{S} \rightarrow\left(\Omega_{S}\right)^{*} \simeq \operatorname{Der}_{S}, \tilde{I}^{*}(\omega):=\tilde{I}^{*}(\omega, \cdot) \text { for } \omega \in \Omega_{S}
\end{aligned}
$$

where $\tilde{I} \circ \tilde{I}^{*}$ and $\tilde{I}^{*} \circ \tilde{I}$ are identities.

Note that $\tilde{I}$ and $\tilde{I}^{*}$ are homogeneous of degree 2 and -2 respectively in the natural sense.

(4.2) For later purposes, we introduce a notation. Let $M$ be an $R$-module. We shall denote by $M\left[\Delta^{-2}\right]$ the localization $M \otimes_{R} R_{\Delta^{2}}$ of $M$ by $\Delta^{2}$.

(4.3) Remember that we have an inclusion $R \subset S$ so that $S$ is finite over $R$.

Then we obtain liftings of forms and vector fields,

$$
\begin{aligned}
& \Omega_{R} \subset \Omega_{S}, d P_{i} \mapsto \sum_{j=1}^{\ell} \frac{\partial P_{i}}{\partial X_{j}} d X_{j}, i=1, \cdots, \ell . \\
& \operatorname{Der}_{R} \subset \operatorname{Der}_{S}\left[\Delta^{-2}\right], \frac{\partial}{\partial P_{i}} \mapsto \sum_{j=1}^{\ell} \frac{\partial X_{j}}{\partial P_{i}} \frac{\partial}{\partial X_{j}}, i=1, \cdots, \ell,
\end{aligned}
$$

which are injective $R$-homomorphisms.

Combining these liftings with the bilinear forms $\tilde{I}$ and $\tilde{I}^{*}$ of (4.1), we obtain symmetric $R$-bilinear forms, which we denote by the same notations $\tilde{I}$ and $\tilde{I}^{*}$.

$$
\begin{aligned}
& \tilde{I}: \operatorname{Der}_{R} \times \operatorname{Der}_{R} \rightarrow R\left[\Delta^{-2}\right] \\
& \tilde{I}^{*}: \Omega_{R} \times \Omega_{R} \rightarrow R
\end{aligned}
$$

$\left(\because\right.$ Since $\tilde{I}$ and $\tilde{I}^{*}$ are $W$-invariant, the values of $\left.\tilde{I}\right|_{\operatorname{Der}_{R} \times \operatorname{Der}_{R}}$ and $\left.\tilde{I}^{*}\right|_{\Omega_{R} \times \Omega_{R}}$ are $W$-invariant.)

(4.4) Now we introduce the concepts of logarithmic one forms and logarithmic vector fields along $\Delta$ and $\Delta^{2}$.

\section{Definition.}

i) $\Omega_{S}(\log \Delta):=\left\{\omega \in \Omega_{S}\left[\Delta^{-2}\right]: \Delta \omega \in \Omega_{S}, d \Delta \wedge \omega \in \Omega_{S} \wedge \Omega_{S}\right\}$ 
$\operatorname{Der}_{S}(\log \Delta):=\left\{\delta \in \operatorname{Der}_{\mathbf{S}}: \delta \Delta \in \Delta S\right\}$

ii) $\Omega_{R}\left(\log \Delta^{2}\right):=\left\{\omega \in \Omega_{R}\left[\Delta^{-2}\right]: \Delta^{2} \omega \in \Omega_{R}, d \Delta^{2} \wedge \omega \in \Omega_{R} \wedge \Omega_{R}\right\}$

$\operatorname{Der}_{R}\left(\log \Delta^{2}\right):=\left\{\delta \in \operatorname{Der}_{R}: \delta \Delta^{2} \in \Delta^{2} R\right\}$

(4.5) The general theory of the modules of logarithmic forms and logarithmic vector fields (cf. [8] §1) shows that the canonical pairing of one forms and vector fields induces non-degenerate $R$ - or $S$-bilinear mappings,

$$
\begin{aligned}
& \Omega_{S}(\log \Delta) \times \operatorname{Der}_{S}(\log \Delta) \rightarrow S \\
& \Omega_{R}\left(\log \Delta^{2}\right) \times \operatorname{Der}_{R}\left(\log \Delta^{2}\right) \rightarrow R
\end{aligned}
$$

so that each of the modules is a dual $S$ - or $R$-module of the other.

(4.6) Theorem. The R-bilinear forms $\tilde{I}$ and $\tilde{I}^{*}$ of (4.3) induce the following non-degenerate $R$-bilinear forms,

i) I: $\operatorname{Der}_{R} \times \operatorname{Der}_{R}\left(\log \Delta^{2}\right) \rightarrow R$

$\tilde{I}^{*}: \Omega_{R} \times \Omega_{R}\left(\log \Delta^{2}\right) \rightarrow R$

so that we obtain R-isomorphisms

ii) I: $\operatorname{Der}_{R} \simeq \Omega_{R}\left(\log \Delta^{2}\right)$

$\tilde{I}^{*}: \Omega_{R} \simeq \operatorname{Der}_{R}\left(\log \Delta^{2}\right)$.

Hence we obtain formulas

iii) $\Omega_{R}\left(\log \Delta^{2}\right)=\tilde{I}(\mathscr{G}) \otimes_{T} R$

$\operatorname{Der}_{R}\left(\log \Delta^{2}\right)=\tilde{I}^{*}(\mathscr{F}) \otimes_{T} R$

iv) $\Omega_{S}(\log \Delta)=\tilde{I}(\mathscr{G}) \otimes_{T} S$

$\operatorname{Der}_{S}(\log \Delta)=\tilde{I}^{*}(\mathscr{F}) \otimes_{T} S$.

Proof. We divide the proof of the theorem (4.6) in several steps.

Step 1. $\tilde{I}^{*} \Omega_{R} \subset \operatorname{Der}_{S}(\log \Delta), \tilde{I}^{*} \Omega_{R} \subset \operatorname{Der}_{R}\left(\log \Delta^{2}\right)$

$\because$ For any $\omega \in \Omega_{R}, I^{*} \omega=I^{*}(\omega, \cdot)$ is a derivation in $\operatorname{Der}_{R}$ and $\operatorname{Der}_{s}$. By definition $\tilde{I}^{*} \omega(\Delta)=\tilde{I}^{*}(\omega, d \Delta)$. Since $\omega$ and $\tilde{I}^{*}$ are $W$-invariant and $\Delta$ is $W$-anti-invariant, $I^{*}(\omega, d \Delta)$ is an anti-invariant, so that $I^{*}(\omega, d \Delta) \in \Delta R$ by (1) viii) a).

Then $\quad \tilde{I}^{*} \omega(\Delta)=\tilde{I}^{*}(\omega, d \Delta) \in \Delta R \subset \Delta S$

and $\quad \tilde{I}^{*} \omega\left(\Delta^{2}\right)=2 \Delta \tilde{I}^{*}(\omega, d \Delta) \in \Delta^{2} R$. 
This means by definition $\tilde{I}^{*} \omega \in \operatorname{Der}_{S}(\log \Delta)$ and $\tilde{I}^{*} \omega \in \operatorname{Der}_{R}\left(\log \Delta^{2}\right)$.

Step 2. Let $\omega_{1}, \cdots, \omega_{\ell}$ be free basis of the $T$-module $\mathscr{F} \subset \Omega_{R}$.

Then $\tilde{I}^{*} \omega_{1}, \cdots, \tilde{I}^{*} \omega_{\ell}$ form free basis of the $S$-module $\operatorname{Der}_{S}(\log \Delta)$ and the $R$-module $\operatorname{Der}_{R}\left(\log \Delta^{2}\right)$.

$\because$ By the use of basis $X_{1}, \cdots, X_{\ell}$ of $V$ and a generator system $P_{1}, \cdots, P_{\ell}$ of $R$, we have presentations,

$$
\tilde{I}^{*} \omega_{i}=\sum_{j=1}^{\ell} \tilde{I}^{*}\left(\omega_{i}, d X_{j}\right) \frac{\partial}{\partial X_{j}} \in \operatorname{Der}_{S}(\log \Delta) \quad i=1, \cdots, \ell
$$

and $\quad \tilde{I}^{*} \omega_{i}=\sum_{j=1}^{\ell} \tilde{I}^{*}\left(\omega_{i}, d P_{j}\right) \frac{\partial}{\partial P_{j}} \in \operatorname{Der}_{R}\left(\log \Delta^{2}\right) \quad i=1, \cdots, \ell$.

Then due the general theory (cf. [8] $\S 1$ (1.5) iii)), it is enough to show, *) $\operatorname{det} .\left(\tilde{I}^{*}\left(\omega_{i}, d X_{j}\right)\right)_{i, j=1, \cdots, \ell}=$ unit $\Delta$

**) $\operatorname{det} .\left(\tilde{I}^{*}\left(\omega_{i}, d P_{j}\right)\right)_{i, j=1, \cdots, \ell}=$ unit $\Delta^{2}$.

Since $*$ ) and $* *$ ) don't depend on the choice of the free basis $\omega_{1}, \cdots, \omega_{\ell}$, we may take $d P_{1}, \cdots, d P_{\ell} \in \mathscr{F}$ as free $T$ basis of $\mathscr{F}$.

Then

$$
\begin{aligned}
\operatorname{det} .\left(\tilde{I}^{*}\left(d P_{i}, d X_{j}\right)\right)_{i, j} & =\operatorname{det} .\left(\sum_{k} \frac{\partial P_{i}}{\partial X_{k}} \tilde{I}^{*}\left(d X_{k}, d X_{j}\right)\right)_{i, j} \\
& =c_{1} \operatorname{det} \frac{\partial\left(P_{1}, \cdots, P_{\ell}\right)}{\partial\left(X_{1}, \cdots, X_{\ell}\right)}
\end{aligned}
$$

and

$$
\begin{aligned}
\operatorname{det} .\left(\tilde{I}^{*}\left(d P_{i}, d P_{j}\right)\right)_{i, j} & =\operatorname{det} .\left(\sum_{k, m} \frac{\partial P_{i}}{\partial X_{k}} \frac{\partial P_{j}}{\partial X_{m}} \tilde{I}\left(d X_{k}, d X_{m}\right)\right)_{i, j} \\
& =c_{2}\left(\operatorname{det} \frac{\partial\left(P_{1}, \cdots, P_{\ell}\right)}{\partial\left(X_{1}, \cdots, X_{\ell}\right)}\right)^{2}
\end{aligned}
$$

where $c_{1}$ and $c_{2}$ are non-zero constants. Thus from (1) viii) b), we obtain the results.

q.e.d.

Step 3. Combining Step 2 with (2.3) ii), we obtain,

$$
\operatorname{Der}_{S}\left(\log \Delta^{2}\right) \simeq \tilde{I}^{*}(\mathscr{F}) \otimes_{T} R \simeq \tilde{I}^{*}\left(\mathscr{F} \bigotimes_{T} R\right) \simeq \tilde{I}^{*}\left(\Omega_{R}\right)
$$


Step 4. Combining Step 3 with (4.5), we obtain,

$$
\begin{aligned}
\mathscr{G} \bigotimes_{T} R & =\operatorname{Der}_{R} \underset{\widetilde{I}}{\widetilde{I}} \tilde{I}\left(\operatorname{Der}_{R}\right)=\tilde{I}\left(\Omega_{R}^{*}\right)=\left(\tilde{I}^{*}\left(\Omega_{R}\right)\right)^{*} \\
& =\left(\operatorname{Der}_{R}\left(\log \Delta^{2}\right)\right)^{*}=\Omega_{R}\left(\log \Delta^{2}\right)
\end{aligned}
$$

Step 5. Proof of iv) of Theorem (4.6).

Using Step 2, we calculate,

$$
\begin{aligned}
\operatorname{Der}_{S}(\log \Delta)=\tilde{I}^{*}(\mathscr{F}) \otimes_{T} S & =\tilde{I}^{*}(\mathscr{F}) \otimes_{T} R \otimes_{R} S \\
& =\operatorname{Der}_{R}\left(\log \Delta^{2}\right) \bigotimes_{R} S
\end{aligned}
$$

Since $\mathscr{F}$ and $\mathscr{G}$ are dual $T$-modules ((2.3) iv)), $\tilde{I}^{*} \mathscr{F}$ and $\tilde{I} \mathscr{G}$ are dual $T$-modules. Thus

$$
\Omega_{R}\left(\log \Delta^{2}\right)=\left(\operatorname{Der}_{R}\left(\log \Delta^{2}\right)\right)^{*}=\left(\tilde{I}^{*} \mathscr{F} \otimes_{T} R\right)^{*}=\tilde{I} \mathscr{G} \otimes_{T} R
$$

and

$$
\begin{aligned}
\Omega_{S}(\log \Delta) & =\left(\operatorname{Der}_{S}(\log \Delta)\right)^{*}=\left(\tilde{I}^{*} \mathscr{F} \otimes_{T} S\right)^{*}=\tilde{I} \mathscr{G} \bigotimes_{T} S \\
& =\tilde{I} \mathscr{G} \otimes_{T} R \otimes_{R} S=\Omega_{R}\left(\log \Delta^{2}\right) \bigotimes_{R} S .
\end{aligned}
$$

This completes the proof of Theorem (4.6).

(4.7) Theorem (4.6) means that the identifications by $\tilde{I}$ and $\tilde{I}^{*}$ induce the following commutative diagrams.

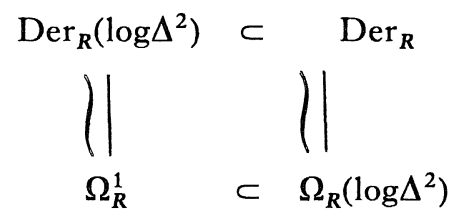

and

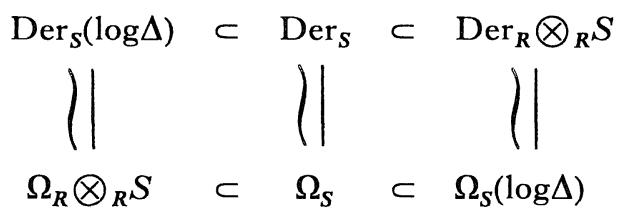

(4.8) Note. Let $\omega_{1}, \cdots, \omega_{\ell}$ be a homogeneous free basis of the $T$-module 
$\mathscr{F}$. We may assume, deg. $\omega_{i}=m_{i}+1, i=1, \cdots, \ell((1)$ ix) and (2.3) ii)).

Then the $S$-free basis $\tilde{I}^{*}\left(\omega_{1}\right), \cdots, \tilde{I}^{*}\left(\omega_{\ell}\right)$ of $\operatorname{Der}_{S}(\log \Delta)$ consists of homogeneous elements with deg. $\tilde{I}^{*}\left(\omega_{i}\right)=\operatorname{deg} . \omega_{i}-2=m_{i}-1, i=1, \cdots, \ell$.

Then vii) and iv) of $\S 1$, imply the following equalities

a) $\sum_{i=1}^{\ell}\left(\operatorname{deg} . \tilde{I}^{*}\left(\omega_{i}\right)+1\right)=\operatorname{deg} . \Delta$

b) $\prod_{i=1}^{\ell}\left(\operatorname{deg} \cdot \tilde{I}^{*}\left(\omega_{i}\right)+2\right)=\# W=$ number of chambers of $W$.

These equalities are those which appeared in the general theory of arrangements (cf. [8] (1.12)).

\section{§5. Bilinear Form $\tilde{J}: \operatorname{Der}_{R} \times \operatorname{Der}_{R} \rightarrow R$}

In this paragraph we introduce a non-degenerate bilinear form $\tilde{J}$ on the tangent bundle of SpecR.

(5.1) Lemma. i) Let us restrict the $R$-bilinear map $\tilde{I}^{*}: \Omega_{R} \times \Omega_{R} \rightarrow R$ to the T-submodule $\mathscr{F} \times \mathscr{F}$. Then the values of $\left.\tilde{I}^{*}\right|_{\mathscr{F} \times \mathscr{F}}$ are polynomial in $P_{\ell}$ of degree one.

ii) Let us define,

$$
J^{*}\left(\omega_{1}, \omega_{2}\right):=D^{(h)} \tilde{I}^{*}\left(\omega_{1}, \omega_{2}\right) \in T \text { for } \omega_{1}, \omega_{2} \in \mathscr{F} .
$$

Then the T-bilinear symmetric map

$$
J^{*}: \mathscr{F} \times \mathscr{F} \rightarrow T
$$

is non-degenerate.

Proof. i) Let $d P_{1}, \cdots, d P_{\ell}$ be a $T$-basis of $\mathscr{F}$. Then $\operatorname{deg} . \tilde{I}^{*}\left(d P_{i}, d P_{j}\right)=$ $\operatorname{deg} . P_{i}+\operatorname{deg} . P_{j}-2<2 h=2$ deg. $P_{\ell}$ for $i, j=1, \cdots, \ell$. Hence as a polynomial in $T\left[P_{\ell}\right], \tilde{I}^{*}\left(d P_{i}, d P_{j}\right)$ is at most of degree one in $P_{\ell}$. Hence $D^{(h)} \tilde{I}^{*}\left(d P_{i}, d P_{j}\right) \in T$.

ii) As in Step 2 of the proof of (4.6), we have,

*) $\quad \operatorname{det} .\left(\left(\tilde{I}^{*}\left(d P_{i}, d P_{j}\right)_{i, j}\right)=\Delta^{2}=c P_{\ell}^{\ell}+A_{1} P_{\ell}^{\ell-1}+\cdots+A_{\ell}\right.$,

where $c$ is a non-zero constant and $A_{i} \in T$, deg. $A_{i}=h i$ (cf. (3.1)).

By comparing the leading coefficients of the two sides of the equality $*$ ), 
we get,

$$
\operatorname{det} .\left(D^{(h)} \tilde{I}^{*}\left(d P_{i}, d P_{j}\right)_{i, j}\right)=c \neq 0 \text {. }
$$

This means that the bilinear mapping $\tilde{J}^{*}=D^{(h)} \tilde{I}^{*}$ is non-degenerate.

q.e.d.

(5.2) Since the $T$-bilinear map $\tilde{J}^{*}: \mathscr{F} \times \mathscr{F} \rightarrow T$ is non-degenerate, we get an isomorphism from $\mathscr{F}$ to its $T$-dual space $\mathscr{F}^{*}=\mathscr{G}$, which we denote by the same symbol $\tilde{J}^{*}$.

$$
\tilde{J}^{*}: \mathscr{F} \stackrel{\sim}{\rightarrow} \mathscr{G}
$$

Then the bilinear form $\widetilde{J}^{*}$ on $\mathscr{F}$ is transformed to a non-degenerate bilinear form $\tilde{J}$ on $\mathscr{G}$,

$$
\tilde{J}: \mathscr{G} \times \mathscr{G} \rightarrow T, \tilde{J}\left(\delta_{1}, \delta_{2}\right)=\left(\tilde{J}^{*}\left(\tilde{J}^{*-1} \delta_{1}, \tilde{J}^{*-1} \delta_{2}\right) \text { for } \delta_{1}, \delta_{2} \in \mathscr{G} .\right.
$$

We shall denote the inverse $\left(\tilde{J}^{*}\right)^{-1}$ by the symbol $\tilde{J}: \mathscr{G} \stackrel{\sim}{\rightarrow} \mathscr{F}$, so that $\tilde{J}^{*}$ and $\tilde{J}^{*} \tilde{J}$ are identities.

Note that $\operatorname{deg} . \tilde{J}^{*}=-(h+2)$ and $\operatorname{deg} . \tilde{J}=\mathrm{h}+2$.

(5.3) Lemma. Let $\left\{\mathscr{F}^{\left(m_{i}+1\right)}\right\}_{i=1, \ldots, \ell}$ and $\left\{\mathscr{G}^{\left(m_{i}+1\right)}\right\}_{i=1, \ldots, \ell}$ be the filtrations of $\mathscr{F}$ and $\mathscr{G}$ introduced in (2.4)

i) $\left.\tilde{J}^{*}\right|_{\mathscr{F}^{(d)} \times \mathscr{F}^{\left(d^{\prime}\right)}}=0 \quad$ for $d+d^{\prime}<h+2$

$$
\left.\widetilde{J}\right|_{\mathscr{G}^{(d)} \times \mathscr{G}^{\left(d^{\prime}\right)}}=0 \quad \text { for } d+d^{\prime}>h+2
$$

ii) $\tilde{J}^{*}: \mathscr{F}^{(d)} \simeq \mathscr{G}^{(h+2-d)}$

$$
\tilde{J}: \mathscr{G}^{(d)} \simeq \mathscr{F}^{(h+2-d)}
$$

Proof. i) Let $P_{1}, \cdots, P_{\ell} \in R$ be a generator system of the algebra R. Then the $d P_{i}, m_{i}+1 \leq d$ form a $T$-basis of $\mathscr{F}^{(d)}$ and the $\frac{\partial}{\partial P_{i}}, m_{i}+1 \geq d$ form a $T$-basis of $\mathscr{G}^{(d)}$.

Evidently, $\widetilde{J}^{*}\left(d P_{i}, d P_{j}\right)=0$ for $m_{i}+m_{j}<h$ and $\widetilde{J}\left(\frac{\partial}{\partial P_{i}}, \frac{\partial}{\partial P_{j}}\right)=0$ for $m_{i}+m_{j}>h$, since the degrees of them are negative. 
ii) Remember (2.6) ii). Then the above i) of (5.3) implies that the image of $\mathscr{F}^{(d)}$ by the morphism $\tilde{J}^{*}$ is contained in $\left(\mathscr{F}^{(h+2-d)^{-}}\right)^{\perp} \cong \mathscr{G}^{(h+2-d)}$. Thus $\tilde{J}^{*}: \mathscr{F} \rightarrow \mathscr{G}$ is an anti-graded homomorphism. We have that $\operatorname{rank}_{T}\left(\mathscr{F}^{(d)}\right)=$ $\#\left\{1 \leq i \leq \ell ; m_{i}+1 \leq d\right\}$ is equal to $\operatorname{rank}_{T}\left(\mathscr{G}^{(h+2-d)}\right)=\left\{1 \leq i \leq \ell: h+2-d \leq m_{i}+1\right\}$ $\left(\because(2.5)\right.$ ii) and (1) ix)). Since $\tilde{J}^{*}$ is non-degenerate, $\tilde{J}^{*}: \mathscr{F}^{(d)} \simeq \mathscr{G}^{(h+2-d)}$. q.e.d.

(5.4) Since we have $R$-isomorphisms: $\operatorname{Der}_{R} \simeq \mathscr{G} \otimes_{T} R$ and $\Omega_{R} \simeq \mathscr{F} \otimes_{T} R$, we may extend $\tilde{J}$ and $\tilde{J}^{*}, R$-linearly to $\operatorname{Der}_{R}$ and $\Omega_{R}$. We shall denote them by the same notations $\tilde{J}$ and $\tilde{J}^{*}$.

$$
\begin{aligned}
& \tilde{J}: \operatorname{Der}_{R} \times \operatorname{Der}_{R} \rightarrow R \\
& \tilde{J}^{*}: \Omega_{R} \times \Omega_{R} \rightarrow R
\end{aligned}
$$

and

$$
\begin{aligned}
& \tilde{J}: \operatorname{Der}_{R} \stackrel{\sim}{\rightarrow} \Omega_{R} \\
& \tilde{J}^{*}: \Omega_{R} \stackrel{\sim}{\rightarrow} \operatorname{Der}_{R}
\end{aligned}
$$

\section{§6. The Grading of $\operatorname{Der}_{R}$ and $\Omega_{R}$ by the Order of $P_{\ell}, v$ and $w$}

From the presentations $\operatorname{Der}_{R} \simeq \mathscr{G} \otimes_{T} T\left[P_{\ell}\right]$ and $\Omega_{R} \simeq \mathscr{F} \otimes_{T} T\left[P_{\ell}\right], \operatorname{Der}_{R}$ and $\Omega_{R}$ have filtrations by the polynomial degree in $P_{\ell}$. In this paragraph, we introduce a somewhat finer structure in $\operatorname{Der}_{R}=\underset{k \geq 0}{\bigoplus} \mathscr{G}_{k}$ and $\Omega_{R}=\underset{k \geq 0}{\bigoplus} \mathscr{F}_{k}$ for later purposes.

(6.1) Lemma. As T-modules, we have the following direct-sum decompositions.

i) $\quad \operatorname{Der}_{R} \simeq \mathscr{G} \oplus \operatorname{Der}_{R}\left(\log \Delta^{2}\right)=\mathscr{G} \oplus \tilde{I}^{*}(\mathscr{F}) \otimes_{T} R$

ii) $\Omega_{R}\left(\log \Delta^{2}\right) \simeq \widetilde{I}(\mathscr{G}) \oplus \Omega_{R}=\tilde{I}(\mathscr{G}) \oplus \mathscr{F} \otimes_{T} R$

Proof. Because of Theorem (4.6) ii), the above statements i) and ii) are equivalent.

Firstly let us show that $\mathscr{G} \cap \tilde{I}^{*}\left(\Omega_{R}\right)=\{0\}$.

Let $\omega \in \Omega_{R}$ satisfy $\tilde{I}^{*}(\omega) \in \mathscr{G}$. By definition of $\mathscr{G}$, this means $\left[D^{(h)}\right.$, $\left.\tilde{I}^{*} \omega\right]=0$. Hence for any $P_{i} \in R, i=1, \cdots, \ell$ 


$$
\begin{aligned}
0=\left[D^{(h)}, \tilde{I}^{*} \omega\right] P_{j} & =D^{(h)} \tilde{I}^{*}\left(\omega, d P_{j}\right)-\tilde{I}^{*}\left(\omega, d D^{(h)} P_{j}\right) \\
& =D^{(h)} \tilde{I}^{*}\left(\omega, d P_{j}\right) .
\end{aligned}
$$

Put $\omega=\sum_{i=1}^{\ell} \varphi^{i} d P_{i}$, where $\varphi^{i} \in T\left[P_{\ell}\right], i=1, \cdots, \ell$ are polynomials in $P_{\ell}$ of degree less than or equal to $d \geq 0$. Then,

$$
\begin{aligned}
0 & =\left(D^{(h)}\right)^{d+1} \tilde{I}^{*}\left(\omega, d P_{j}\right)=\left(D^{(h)}\right)^{d+1} \sum_{i=1}^{\ell} \tilde{I}^{*}\left(\varphi^{i} d P_{i}, d P_{j}\right) \\
& =\sum_{i=1}^{\ell} \sum_{k=0}^{d+1} C_{k}^{d+1}\left(\left(D^{(h)}\right)^{d+1-k} \varphi^{i}\right)\left(D^{(h)}\right)^{k} \tilde{I}^{*}\left(d P_{i}, d P_{j}\right) \\
& =\sum_{i=1}^{\ell} C_{1}^{d+1}\left(\left(D^{(h)}\right)^{d} \varphi^{i}\right) \tilde{J}^{*}\left(d P_{i}, d P_{j}\right)
\end{aligned}
$$

Since $\left(\tilde{J}^{*}\left(d P_{i}, d P_{j}\right)\right)_{i, j=1, \cdots, \ell}$ is non-degenerate $\left((5.1)\right.$ ii)), we get $\left(D^{(h)}\right)^{d} \varphi^{i}=0$ $i=1, \cdots, \ell$.

By descending induction on $d$, we conclude $\varphi^{i}=0, i=1, \cdots, \ell$.

Conversely let us show that $\operatorname{Der}_{R} \subset \mathscr{G}+\tilde{I}^{*}(\mathscr{F})\left[P_{\ell}\right]$.

Take any element $\delta=\sum_{i=1}^{\ell} \varphi_{i} \frac{\partial}{\partial P_{i}}$ of $\operatorname{Der}_{R}$ where $\varphi_{i} \in T\left[P_{\ell}\right], \mathrm{i}=1, \cdots, \ell$ are polynomial in $P_{\ell}$ of degree $d$. If $d=0$, then $\delta \in \mathscr{G}$. Suppose $d>0$ and let us show the assertion by induction on $d$.

Let us decompose, $\varphi_{i}=\psi_{i}+a_{i} P_{\ell}^{d}, i=1, \cdots, \ell$, where $a_{i} \in T i=1, \cdots, \ell$ and $\psi_{1}, \cdots, \psi_{\ell} \in T\left[P_{\ell}\right]$ are polynomials in $P_{\ell}$ of degree less than $d$. Since $\left(\tilde{J}^{*}\left(d P_{i}, d P_{j}\right)\right)_{i, j=1, \cdots, \ell}$ is non-degenerate, one can find $b^{1}, \cdots, b^{\ell} \in T$, such that $a_{j}=\sum_{i=1}^{\ell} b^{i} D^{(h)} \tilde{I}^{*}\left(d P_{i}, d P_{j}\right), j=1, \cdots, \ell . \quad$ Put $\delta^{\prime}=\sum_{i=1}^{\ell} b^{i} P_{\ell}^{d-1} \tilde{I}^{*}\left(d P_{i}\right) . \quad$ Then $\delta^{\prime} \in$ $\tilde{I}^{*}(\mathscr{F})\left[P_{\ell}\right] . \quad$ Since $\delta^{\prime}=\sum_{i, j} b^{i} \widetilde{I}^{*}\left(d P_{i}, d P_{j}\right) P_{\ell}^{d-1} \frac{\partial}{\partial P_{j}}$, the coefficients of $\delta-\delta^{\prime}$ are of degree less than $d$ in $P_{\ell}$.

This completes the proof of Lemma (6.1).

(6.2) Let us introduce two endomorphisms of $\operatorname{Der}_{R}$.

$$
\begin{aligned}
& v: \operatorname{Der}_{R} \rightarrow \operatorname{Der}_{R}, \quad \delta \mapsto\left[D^{(h)}, \delta\right] \quad(T \text {-homomorphism) } \\
& w: \operatorname{Der}_{R} \rightarrow \operatorname{Der}_{R}, \quad \delta \mapsto\left(\tilde{I}^{*} \circ \widetilde{J}\right) \delta \quad \text { (R-homomorphism) }
\end{aligned}
$$

Then we have the commutator relation, $[v, w]=$ identity. 
Proof. For an element $\delta \in \sum_{i=1}^{\ell} \varphi_{i} \frac{\partial}{\partial P_{i}}$ of $\operatorname{Der}_{R}$,

$$
\begin{aligned}
v(w(\delta)) & =\left[D^{(h)}, \tilde{I}^{*} \widetilde{J}(\delta)\right]=\left[D^{(h)}, \sum_{i} \varphi_{i} \tilde{I}^{*} \widetilde{J}\left(\frac{\partial}{\partial P_{i}}\right)\right] \\
& =\sum_{i}\left(D^{(h)} \varphi_{i}\right) \tilde{I}^{*} \widetilde{J}\left(\frac{\partial}{\partial P_{i}}\right)+\sum_{i} \varphi_{i}\left[D^{(h)}, \tilde{I}^{*} \tilde{J} \frac{\partial}{\partial P_{i}}\right] \\
& =\tilde{I}^{*} \widetilde{J}\left(\left[D^{(h)}, \delta\right]\right)+\sum_{i} \varphi_{i} \tilde{J}^{*} \tilde{J} \frac{\partial}{\partial P_{i}} \\
& =w(v(\delta))+\delta
\end{aligned}
$$

q.e.d.

(6.3) Put $\quad \mathscr{G}_{0}:=\mathscr{G}$ and $\mathscr{G}_{k}:=w^{k} \mathscr{G}_{0} \quad k \in N$

$$
\mathscr{F}_{0}:=\mathscr{F} \text { and } \mathscr{F}_{k}:=\left(\tilde{J}_{\circ} \tilde{I}^{*}\right)^{k} \mathscr{F}_{0} \quad k \in N
$$

i) As T-modules, we have the following direct sum decompositions.

$$
\begin{array}{ll}
\operatorname{Der}_{R}=\bigoplus_{k=0}^{\infty} \mathscr{G}_{k}, & \operatorname{Der}_{R}\left(\log \Delta^{2}\right)=\bigoplus_{k=1}^{\infty} \mathscr{G}_{k} \\
\Omega_{R}=\bigoplus_{k=0}^{\infty} \mathscr{F}_{k}, & \Omega_{R}\left(\log \Delta^{2}\right)=\bigoplus_{k=-1}^{\infty} \mathscr{F}_{k} .
\end{array}
$$

ii) Let us denote by $R_{N}$ the $T$-submodule of $R$ of all polynomials of $P_{\ell}$ of degree less than or equal to $N$. Then

$$
\begin{aligned}
& \mathscr{G}_{k} \oplus \mathscr{G}_{k+1} \oplus \cdots \oplus \mathscr{G}_{k+N} \simeq \mathscr{G}_{k} \otimes_{T} R_{N} \\
& \mathscr{F}_{k} \oplus \mathscr{F}_{k+1} \oplus \cdots \oplus \mathscr{F}_{k+N} \simeq \mathscr{F}_{k} \otimes_{T} R_{N}
\end{aligned}
$$

Proof. We shall prove the statements on $\mathscr{G}_{*}$. The statements on $\mathscr{F}_{*}$ are shown almost similarly by using Lie derivatives $L_{D^{(h)}}$ and $\widetilde{J}_{\circ} \widetilde{I}^{*}$ instead of $v$ and $w$ so that we omit them.

By repeated use of (6.1), we get decompositions,

$$
\operatorname{Der}_{R}=\bigoplus_{k=0}^{N} \mathscr{G}_{k} \oplus w^{N+1}\left(\operatorname{Der}_{R}\right)
$$




$$
\operatorname{Der}_{R}\left(\log \Delta^{2}\right)=\bigoplus_{k=1}^{N} \mathscr{G}_{k} \oplus w^{N+1}\left(\operatorname{Der}_{R}\left(\log \Delta^{2}\right)\right)
$$

Thus $\operatorname{Der}_{R} \supset \bigoplus_{k=0}^{\infty} \mathscr{G}_{k}$ and $\operatorname{Der}_{R}\left(\log \Delta^{2}\right) \supset \bigoplus_{k=1}^{\infty} \mathscr{G}_{k}$.

To show the converse inclusions, it is enough to show ii) of (6.3). By shifting by $w^{k}$, we have only to show, $\mathscr{G}_{0} \oplus \cdots \oplus \mathscr{G}_{N}=\mathscr{G}_{0} \otimes_{T} R_{N}$.

Assertion. $\left.\quad v\right|_{\mathscr{G}_{k}}=k\left(\left.w\right|_{\mathscr{G}_{k-1}}\right)^{-1}$.

Proof. Let us show the assertion by induction on $k$. For $k=0$, it is trivial. For an $\delta \in \mathscr{G}_{k-1}$,

$$
\begin{aligned}
v(w(\delta)) & =w(v(\delta))+\delta \quad(\because(6.2)) \\
& =w\left((k-1) w^{-1}(\delta)\right)+\delta=k \delta
\end{aligned}
$$

(By induction hypothesis)

As a corollary of the above assertion, $\left.v^{k+1}\right|_{\mathscr{G}_{k}}=0$. Hence $\mathscr{G}_{0} \oplus \cdots \oplus$ $\mathscr{G}_{N} \subseteq \operatorname{ker}\left(v^{N+1}\right)=\mathscr{G}_{0} \otimes R_{N}$. Conversely take an element $\delta$ of $\operatorname{ker}\left(v^{N+1}\right)$. Since $v^{N+1} \delta=0, v^{N} \delta$ is contained in $\mathscr{G}=\mathscr{G}_{0}$. Put $\delta^{\prime}=\delta-(N !)^{-1} w^{N} v^{N} \delta$. Then by a repeated use of the above assertion, $v^{N}\left(\delta^{\prime}\right)=v^{N} \delta-(N !)^{-1} v^{N} w^{N} v^{N} \delta=$ 0 . Hence $\delta=\delta^{\prime}+(N !)^{-1} w^{N} v^{N} \delta \in \operatorname{ker} v^{N}+\mathscr{G}_{N}$. By induction on $N$, this proves the ii) of (6.3).

q.e.d.

$(6.4)$ i) For any $\delta \in \mathscr{G}, w^{k}(\delta)$ is the last direct sum factor of $P_{\ell}^{k} \delta \in \mathscr{G}_{0} \oplus \cdots \oplus$ $\mathscr{G}_{k}$.

ii) $\left[w^{k}(\delta), w^{m}\left(\delta^{\prime}\right)\right]=w^{k+m}\left(\left[\delta, \delta^{\prime}\right]\right) \bmod \mathscr{G}_{0} \oplus \cdots \oplus \mathscr{G}_{k+m-1}$, for $\delta, \delta^{\prime} \in \mathscr{G}$.

Proof. By ii) of (6.3) $P_{\ell}^{k} \delta \in \mathscr{G}_{0} \oplus \cdots \oplus \mathscr{G}_{k}$. Using the assertion of the proof of (6.3), we compute $v^{k}\left(P_{\ell}^{k} \delta-\mathrm{w}^{k} \delta\right)=k ! \delta-k ! \delta=0$. Hence $P_{\ell}^{k} \delta-w^{k} \delta$ $\epsilon \operatorname{ker} v^{k}=\mathscr{G}_{0} \oplus \cdots \oplus \mathscr{G}_{k-1}$.

(6.5) By definition of w (6.2), we have a formula,

$$
\widetilde{J}\left(\delta, \delta^{\prime}\right)=\widetilde{I}\left(w \delta, \delta^{\prime}\right)=\tilde{I}\left(\delta, w \delta^{\prime}\right) \quad \text { for } \delta, \delta^{\prime} \in \operatorname{Der}_{R} .
$$

This formula is very interesting, since two bilinear forms $\tilde{J}$ and $\tilde{I}$ are combined by the use of $w$. For instance, suppose we have informations of 
$D^{(h)}$ and $\Delta^{2} \in R$ and $\tilde{J}$ but not of $\tilde{I}$. Then one may reconstruct $\tilde{I}$ as follows.

i) The direct sum decomposition $\operatorname{Der}_{R}=\mathscr{G} \oplus \operatorname{Der}_{R}\left(\log \Delta^{2}\right)$ depends only on $D^{(h)}$ and $\Delta^{2}$.

ii) w: $\mathscr{G}_{0} \rightarrow \mathscr{G}_{1} \subset \operatorname{Der}_{R}\left(\log \Delta^{2}\right)$ is well defined by using only the direct sum decomposition above. i.e. $w(\delta)=$ the second factor of $P_{\ell} \delta \in \mathscr{G} \oplus \operatorname{Der}_{R}\left(\log \Delta^{2}\right)$.

iii) Since $\left.\widetilde{J}\right|_{\mathscr{G} \times \mathscr{G}}$ is non-degenerate, let $\delta_{1}, \cdots, \delta_{\ell} \in \mathscr{G}$ and $\delta^{1 *}, \cdots, \delta^{\ell *} \in \mathscr{G}$ be dual basis of $\mathscr{G}$ with respect to $\tilde{J}$.

iv) Then $\tilde{I}^{*}=\sum_{i=1}^{\ell} \delta_{i} \otimes w \delta^{i *}$.

In the application, $\widetilde{J}$ is described by certain duality of local cohomology groups and $\tilde{I}$ is describing the intersection form of certain vanishing cycles of rational double points. Thus the above construction means that the topological intersection form is described by algebraic local cohomology groups and vice versa. This process is valid not only for rational double points but for all isolated hypersurface singularities. For the detailed study see [7] and (11.4) of the present note (cf. [42]).

\section{$\S 7$. The Euler Operator $E$}

In this paragraph we introduce the concept of an Euler operator $E$.

(7.1) Let I be the W-invariant quadratic form of (1) $\mathrm{x})$. Here we understand $I$ as an element of $R$.

Definition. We denote by $E$ the logarithmic vector field $\tilde{I}^{*}(d I) \in$ $\operatorname{Der}_{R}\left(\log \Delta^{2}\right)$ and call it the Euler operator.

(7.2) (i) The Euler operator $E$ has the following presentations

$$
\begin{aligned}
E & =\sum_{i=1}^{\ell} X_{i} \frac{\partial}{\partial X_{i}} \quad \text { for } \quad X_{1}, \cdots, X_{\ell} \text { basis of } V \\
& \left.=\sum_{i=1}^{\ell}\left(m_{i}+1\right) P_{i} \frac{\partial}{\partial P_{i}} \quad \text { for } \quad P_{1}, \cdots, P_{\ell} \text { a generator of } R \text { in }(1) v\right) .
\end{aligned}
$$

ii) $E P=(\operatorname{deg} . P) P$ for a homogeneous $P \in S$ or $R$.

iii) $[E, \delta]=(\operatorname{deg} . \delta) \delta$ for a homogeneous $\delta \in \operatorname{Der}_{S}$ or $\operatorname{Der}_{R}$, where [ , ] is the bracket product of vector fields. 
iv) $L_{E} \omega=(\operatorname{deg} . \omega) \omega$ for a homogeneous $\omega \in \Omega_{S}$ or $\Omega_{R}$, where $L_{E}$ is the Lie derivative of $E$.

v) Euler operator is equal to $h w\left(D^{(h)}\right)$.

Proof. By definition of $\tilde{I}^{*}, \tilde{I}^{*}(d I)$ is the identity on $V=S^{1}$.

i.e. $E X=\tilde{I}^{*}(d I, d X)=\sum_{j=1}^{\ell} \frac{\partial I}{\partial X_{j}} I^{*}\left(X_{j}, X\right)=X \quad$ for any $X \in V$.

Then as a vector field in $\operatorname{Der}_{S}, E$ has the following development

$$
E=\sum_{i=1}^{\ell}\left(E X_{i}\right) \frac{\partial}{\partial X_{i}}=\sum_{i=1}^{\ell} X_{i} \frac{\partial}{\partial X_{i}}
$$

Therefore as an element of $\operatorname{Der}_{R}, E$ has the development

$$
E=\sum_{i=1}^{\ell}\left(E P_{i}\right) \frac{\partial}{\partial P_{i}}=\sum_{i=1}^{\ell}\left(\operatorname{deg} \cdot P_{i}\right) P_{i} \frac{\partial}{\partial P_{i}}
$$

Then the assertions ii), iii) and iv) are easy consequences of these presentations of $E$.

On the other hand, $\tilde{J}^{*}(d I)=\sum_{j=1}^{\ell} D^{(h)} \tilde{I}^{*}\left(d I, d P_{j}\right) \frac{\partial}{\partial P_{j}}=\sum_{j=1}^{\ell} D^{(h)}\left(E P_{j}\right) \frac{\partial}{\partial P_{j}}=$ $h\left(D^{(h)} P_{\ell}\right) \frac{\partial}{\partial P_{\ell}}=h D^{(h)} . \quad$ Then $h w\left(D^{(h)}\right)=h \tilde{I}^{*} \widetilde{J}\left(D^{(h)}\right)=\tilde{I}^{*}(d I)=E . \quad$ q.e.d.

(7.3) In the presentation $E=h w\left(D^{(h)}\right)$ of (7.2) v), we don't need the information on $\tilde{I}$ or $\tilde{J}$. i.e. The Euler operator can be constructed only by the informations of $D^{(h)}$ and $\Delta^{2} \in R$ (cf. (6.5)).

\section{§. Torsion Free Connection $\nabla$ with $\nabla \tilde{I}^{*}=0$}

In this paragraph we characterize the vector space $V$ by means of a connection $\nabla$ for a later purpose. This whole paragraph may be considered as a preparation for the proof of Theorem (9.4).

(8.1) A torsion free affine connection $\nabla$ is an $\boldsymbol{R}$-bilinear map

$$
\begin{gathered}
\nabla: \operatorname{Der}_{R}\left[\Delta^{-2}\right] \times \operatorname{Der}_{R}\left[\Delta^{-2}\right] \rightarrow \operatorname{Der}_{R}\left[\Delta^{-2}\right] \\
\left(\delta, \delta^{\prime}\right) \mapsto \nabla_{\delta} \delta^{\prime}
\end{gathered}
$$


such that i) $\nabla_{P \delta} \delta^{\prime}=P \nabla_{\delta} \delta^{\prime}$ for $P \in R\left[\Delta^{-2}\right]$

ii) Leibniz rule, $\nabla_{\delta}\left(P \delta^{\prime}\right)=(\delta P) \delta^{\prime}+P \nabla_{\delta} \delta^{\prime}$ for $P \in R\left[\Delta^{-2}\right]$

iii) Torsion free, $\nabla_{\delta} \delta^{\prime}-\nabla_{\delta^{\prime}} \delta=\left[\delta, \delta^{\prime}\right]$

Here, one recalls that the notation $M\left[\Delta^{-2}\right]$ for an $R$-module $M$ means the localization $M \otimes_{R} R_{\Delta^{2}}$ (cf. (4.2)).

(8.2) The next fact is a standard result in Riemannian geometry. For the sake of completeness, we give a proof of it.

There exist uniquely a torsion free affine connection $\nabla$, with the property, $\nabla \tilde{I}^{*}=0$.

i.e. $\quad \delta \tilde{I}\left(\delta^{\prime}, \delta^{\prime \prime}\right)=\tilde{I}\left(\nabla_{\delta} \delta^{\prime}, \delta^{\prime \prime}\right)+\widetilde{I}\left(\delta^{\prime}, \nabla_{\delta} \delta^{\prime \prime}\right) \quad$ for $\delta, \delta^{\prime}, \delta^{\prime \prime} \in \operatorname{Der}_{R}\left[\Delta^{-2}\right]$

(This connection is often called the Levi-Civita or Riemannian connection.)

Proof. i) Uniqueness. Using the torsion freeness and $\nabla \tilde{I}^{*}=0$, we compute

$$
\begin{aligned}
& \widetilde{I}\left(\nabla_{\delta_{1}} \delta_{2}, \delta_{3}\right)=\delta_{1} \widetilde{I}\left(\delta_{2}, \delta_{3}\right)-\widetilde{I}\left(\delta_{2}, \nabla_{\delta_{1}} \delta_{3}\right) \\
& =\delta_{1} \tilde{I}\left(\delta_{2}, \delta_{3}\right)-\tilde{I}\left(\delta_{2},\left[\delta_{1}, \delta_{3}\right]\right)-\tilde{I}\left(\delta_{2}, \nabla_{\delta_{3}} \delta_{1}\right) \\
& =\delta_{1} \tilde{I}\left(\delta_{2}, \delta_{3}\right)-\tilde{I}\left(\delta_{2},\left[\delta_{1}, \delta_{3}\right]\right)-\delta_{3} \tilde{I}\left(\delta_{2}, \delta_{1}\right)+\tilde{I}\left(\nabla_{\delta_{3}} \delta_{2}, \delta_{1}\right) \\
& =\delta_{1} \tilde{I}\left(\delta_{2}, \delta_{3}\right)-\tilde{I}\left(\delta_{2},\left[\delta_{1}, \delta_{3}\right]\right)-\delta_{3} \tilde{I}\left(\delta_{2}, \delta_{1}\right) \\
& +\widetilde{I}\left(\left[\delta_{3}, \delta_{2}\right], \delta_{1}\right)+\widetilde{I}\left(\nabla_{\delta_{2}} \delta_{3}, \delta_{1}\right) \\
& =\delta_{1} \tilde{I}\left(\delta_{2}, \delta_{3}\right)-\tilde{I}\left(\delta_{2},\left[\delta_{1}, \delta_{3}\right]\right)-\delta_{3} \tilde{I}\left(\delta_{2}, \delta_{1}\right)+\widetilde{I}\left(\left[\delta_{3}, \delta_{2}\right], \delta_{1}\right) \\
& +\delta_{2} \tilde{I}\left(\delta_{3}, \delta_{1}\right)-\tilde{I}\left(\delta_{3}, \nabla_{\delta_{2}} \delta_{1}\right) \\
& =\delta_{1} \tilde{I}\left(\delta_{2}, \delta_{3}\right)-\tilde{I}\left(\delta_{2},\left[\delta_{1}, \delta_{3}\right]\right)-\delta_{3} \tilde{I}\left(\delta_{2}, \delta_{1}\right)+\widetilde{I}\left(\left[\delta_{3}, \delta_{2}\right], \delta_{1}\right) \\
& +\delta_{2} \widetilde{I}\left(\delta_{3}, \delta_{1}\right)-\widetilde{I}\left(\delta_{3},\left[\delta_{2}, \delta_{1}\right]\right)-\widetilde{I}\left(\delta_{3}, \nabla_{\delta_{1}} \delta_{2}\right) \text {. }
\end{aligned}
$$

Thus altogether we obtain the FORMULA

i) $2 \tilde{I}\left(\nabla_{\delta_{1}} \delta_{2}, \delta_{3}\right)=\delta_{1} \tilde{I}\left(\delta_{2}, \delta_{3}\right)+\delta_{2} \tilde{I}\left(\delta_{3}, \delta_{1}\right)-\delta_{3} \tilde{I}\left(\delta_{1}, \delta_{2}\right)$

$$
-\tilde{I}\left(\delta_{1},\left[\delta_{2}, \delta_{3}\right]\right)+\tilde{I}\left(\delta_{2},\left[\delta_{3}, \delta_{1}\right]\right)+\tilde{I}\left(\delta_{3},\left[\delta_{1}, \delta_{2}\right]\right) \text {. }
$$

Since the right-hand of i) is calculated only by the use of $\tilde{I}$, the left-hand $\tilde{I}\left(\nabla_{\delta_{1}} \delta_{2}, \delta_{3}\right)$ does not depend on $\nabla$. Since $\tilde{I}$ is non-degenerate over $R\left[\Delta^{-2}\right]$, $\nabla_{\delta_{1}} \delta_{2}$ is uniquely determined by the formula. 
ii) Existence. Let us denote by $f\left(\delta_{1}, \delta_{2}, \delta_{3}\right)$ the right-hand of the formula of i). Then one may check easily the following properties,

i) $f$ is additive for each variable $\delta_{1}, \delta_{2}, \delta_{3}$.

ii) $f\left(P \delta_{1}, \delta_{2}, \delta_{3}\right)=P f\left(\delta_{1}, \delta_{2}, \delta_{3}\right)$

$$
\begin{aligned}
f\left(\delta_{1}, P \delta_{2}, \delta_{3}\right) & =P f\left(\delta_{1}, \delta_{2}, \delta_{3}\right)+2\left(\delta_{1} P\right) \tilde{I}\left(\delta_{2}, \delta_{3}\right) \\
f\left(\delta_{1}, \delta_{2}, P \delta_{3}\right) & =P f\left(\delta_{1}, \delta_{2}, \delta_{3}\right) \\
\text { for } P & \in R\left[\Delta^{-2}\right] \text { and } \delta_{1}, \delta_{2}, \delta_{3} \in \operatorname{Der}_{R}\left[\Delta^{-2}\right]
\end{aligned}
$$

iii) $f\left(\delta_{1}, \delta_{2}, \delta_{3}\right)-f\left(\delta_{2}, \delta_{1}, \delta_{3}\right)=2 \tilde{I}\left(\left[\delta_{1}, \delta_{2}\right], \delta_{3}\right)$

iv) $f\left(\delta_{1}, \delta_{2}, \delta_{3}\right)+f\left(\delta_{1}, \delta_{3}, \delta_{2}\right)=2 \delta_{1} \tilde{I}\left(\delta_{2}, \delta_{3}\right)$.

Then i) and ii) imply that there exists an affine connection $\nabla$ such that $f\left(\delta_{1}, \delta_{2}, \delta_{3}\right)=2 \tilde{I}\left(\nabla_{\delta_{1}} \delta_{2}, \delta_{3}\right)$. The property iii) implies that $\nabla$ is torsion free and iv) implies that $\nabla \tilde{I}^{*}=0$.

\section{(8.3) The connection $\nabla$ is integrable.}

i.e. $\nabla_{\delta_{1}} \nabla_{\delta_{2}}-\nabla_{\delta_{2}} \nabla_{\delta_{1}}=\nabla_{\left[\delta_{1}, \delta_{2}\right]}$ for $\delta_{1}, \delta_{2} \in \operatorname{Der}_{R}\left[\Delta^{-2}\right]$.

Proof. Let us extend the connection $\nabla$ to the module.

$$
\operatorname{Der}_{S}\left[\Delta^{-2}\right] \simeq \operatorname{Der}_{R}\left[\Delta^{-2}\right] \otimes_{R} S
$$

This extension will be done in the following way. Firstly, note that we may extend $\tilde{I}$ to the module $\operatorname{Der}_{S}\left[\Delta^{-2}\right]$

$$
\tilde{I}: \operatorname{Der}_{S}\left[\Delta^{-2}\right] \times \operatorname{Der}_{S}\left[\Delta^{-2}\right] \rightarrow S\left[\Delta^{-2}\right] .
$$

Then as in (8.2) we define,

$$
\begin{aligned}
2 \tilde{I}\left(\tilde{\nabla}_{\delta_{1}} \delta_{2}, \delta_{3}\right)= & \delta_{1} \tilde{I}\left(\delta_{2}, \delta_{3}\right)+\delta_{2} \tilde{I}\left(\delta_{3}, \delta_{1}\right)-\delta_{3} \tilde{I}\left(\delta_{1}, \delta_{2}\right) \\
& -\widetilde{I}\left(\delta_{1}\left[\delta_{2}, \delta_{3}\right]\right)+\tilde{I}\left(\delta_{2},\left[\delta_{3}, \delta_{1}\right]\right)+\tilde{I}\left(\delta_{3},\left[\delta_{1}, \delta_{2}\right]\right) \\
& \quad \text { for } \delta_{1}, \delta_{2}, \delta_{3} \in \operatorname{Der}_{s}\left[\Delta^{-2}\right]
\end{aligned}
$$

so that we obtain a connection,

$$
\tilde{\nabla}: \operatorname{Der}_{S}\left[\Delta^{-2}\right] \times \operatorname{Der}_{S}\left[\Delta^{-2}\right] \rightarrow \operatorname{Der}_{S}\left[\Delta^{-2}\right]
$$

such that $\left.\tilde{\nabla}\right|_{\operatorname{Der}_{R}\left[\Delta^{-2}\right]}=\nabla$. Since $\tilde{\nabla}$ is the Levi-Civita connection for the flat inner product $I$ on the vector space $V, \tilde{\nabla}$ is integrable and hence its 
restriction $\nabla=\left.\tilde{\nabla}\right|_{\operatorname{Der}_{R}\left[\Delta^{-2}\right]}$ is integrable.

q.e.d.

(8.4) The connection $\nabla$ is logarithmic in the following sense

i) $\nabla: \operatorname{Der}_{R}\left(\log \Delta^{2}\right) \times \operatorname{Der}_{R}\left(\log \Delta^{2}\right) \rightarrow \operatorname{Der}_{R}\left(\log \Delta^{2}\right)$.

More precisely, for $i, j \geq 1$,

$\nabla: \mathscr{G}_{1} \oplus \mathscr{G}_{2} \oplus \cdots \oplus \mathscr{G}_{i} \times \mathscr{G}_{1} \oplus \mathscr{G}_{2} \oplus \cdots \oplus \mathscr{G}_{j} \rightarrow \mathscr{G}_{1} \oplus \mathscr{G}_{2} \oplus \cdots \oplus \mathscr{G}_{i+j}$

ii) $\nabla: \operatorname{Der}_{R} \times \operatorname{Der}_{R}\left(\log \Delta^{2}\right) \rightarrow \operatorname{Der}_{R}$.

More precisely, for $i \geq 0, j \geq 1$,

$\nabla: \mathscr{G}_{0} \oplus \mathscr{G}_{1} \oplus \cdots \oplus \mathscr{G}_{i} \times \mathscr{G}_{1} \oplus \mathscr{G}_{2} \oplus \cdots \oplus \mathscr{G}_{j} \rightarrow \mathscr{G}_{0} \oplus \mathscr{G}_{1} \oplus \cdots \oplus \mathscr{G}_{i+j}$

iii) $\nabla: \operatorname{Der}_{R}\left(\log \Delta^{2}\right) \times \operatorname{Der}_{R} \rightarrow \operatorname{Der}_{R}$

More precisely, for $i \geq 1, j \geq 0$,

$\nabla: \mathscr{G}_{1} \oplus \mathscr{G}_{2} \oplus \cdots \oplus \mathscr{G}_{i} \times \mathscr{G}_{0} \oplus \mathscr{G}_{1} \oplus \cdots \oplus \mathscr{G}_{j} \rightarrow \mathscr{G}_{0} \oplus \mathscr{G}_{1} \oplus \cdots \oplus \mathscr{G}_{i+j}$.

Proof. Remember that, $\operatorname{Der}_{R}=\mathscr{G}_{0} \otimes_{T} R$ and $\operatorname{Der}_{R}\left(\log \Delta^{2}\right)=\mathscr{G}_{1} \otimes_{T} R$, and $\mathscr{G}_{0} \oplus \cdots \oplus \mathscr{G}_{i}=\mathscr{G}_{0} \otimes_{T} R_{i}$ and $\mathscr{G}_{1} \oplus \cdots \oplus \mathscr{G}_{i}=\mathscr{G}_{1} \otimes_{T} R_{i-1}$, where $R_{i}$ denotes the $T$-submodule of $R=T\left[P_{\ell}\right]$ of all polynomials in $P_{\ell}$ of degree less or equal than $i$ (cf. (6.3)).

Then by using the Leibniz rule of the connection, one may reduce the proof to the following simplest cases.

i) $\nabla: \mathscr{G}_{1} \times \mathscr{G}_{1} \rightarrow \mathscr{G}_{1} \oplus \mathscr{G}_{2}$

ii) $\nabla: \mathscr{G}_{0} \times \mathscr{G}_{1} \rightarrow \mathscr{G}_{0} \oplus \mathscr{G}_{1}$

iii) $\nabla: \mathscr{G}_{1} \times \mathscr{G}_{0} \rightarrow \mathscr{G}_{0} \oplus \mathscr{G}_{1}$

Proof of i). For $\delta_{1}, \delta_{2}, \delta_{3}$ of $\mathscr{G}_{0}$, let us compute,

$$
\begin{aligned}
\tilde{I}\left(\nabla_{w\left(\delta_{1}\right)} w\left(\delta_{2}\right), \delta_{3}\right) & \\
= & w\left(\delta_{1}\right) \tilde{I}\left(w\left(\delta_{2}\right), \delta_{3}\right)+w\left(\delta_{2}\right) \tilde{I}\left(\delta_{3}, w\left(\delta_{1}\right)\right)-\delta_{3} \tilde{I}\left(w\left(\delta_{1}\right), w\left(\delta_{2}\right)\right) \\
& -\tilde{I}\left(w\left(\delta_{1}\right)\left[w\left(\delta_{2}\right), \delta_{3}\right]\right)+\tilde{I}\left(w\left(\delta_{2}\right),\left[\delta_{3}, w\left(\delta_{1}\right)\right]\right)+\tilde{I}\left(\delta_{3},\left[w\left(\delta_{1}\right), w\left(\delta_{2}\right)\right]\right) \\
= & w\left(\delta_{1}\right) \tilde{J}\left(\delta_{2}, \delta_{3}\right)+w\left(\delta_{2}\right) \tilde{J}\left(\delta_{3}, \delta_{1}\right)-\delta_{3} \widetilde{J}\left(\delta_{1}, w\left(\delta_{2}\right)\right) \\
& -\tilde{J}\left(\delta_{1},\left[w\left(\delta_{2}\right), \delta_{3}\right]\right)+\widetilde{J}\left(\delta_{2},\left[\delta_{3}, w\left(\delta_{1}\right)\right]\right)+\widetilde{J}\left(\delta_{3}, w\left(\left[\delta_{1}, \delta_{2}\right]\right)+\delta^{\prime}\right)
\end{aligned}
$$

where $\left[w\left(\delta_{1}\right), w\left(\delta_{2}\right)\right]=w^{2}\left(\left[\delta_{1}, \delta_{2}\right]\right)+w\left(\delta^{\prime}\right)$ (cf. (6.4) ii)). Thus $\tilde{I}\left(\nabla_{w\left(\delta_{1}\right)} w\left(\delta_{2}\right), \delta_{3}\right)$ $\in R_{1}$ for $\delta_{1}, \delta_{2}, \delta_{3} \in \mathscr{G}_{0}$. 
Denote $\varphi\left(\delta_{3}\right)=\widetilde{I}\left(\nabla_{w\left(\delta_{1}\right)} w\left(\delta_{2}\right), \delta_{3}\right)$ for fix $\delta_{1}, \delta_{2}$. Then $D^{(h)} \varphi\left(\delta_{3}\right) \in T$ for $\delta_{3} \in \mathscr{G}_{0}$. Thus there exist a $\delta_{4} \in \mathscr{G}_{0}$, such that $D^{(h)} \varphi\left(\delta_{3}\right)=\widetilde{J}\left(\delta_{4}, \delta_{3}\right)$ (cf. (5.2)). Thus $\varphi\left(\delta_{3}\right)-\widetilde{I}\left(w^{2}\left(\delta_{4}\right), \delta_{3}\right) \in T$ for $\delta_{3} \in \mathscr{G}_{0}$. Then there exist a $\delta_{5} \in \mathscr{G}_{0}$ such that $\varphi\left(\delta_{3}\right)-\widetilde{I}\left(w^{2}\left(\delta_{4}\right), \delta_{3}\right)=\widetilde{J}\left(\delta_{5}, \delta_{3}\right)=\widetilde{I}\left(w\left(\delta_{5}\right), \delta_{3}\right)$. Hence $\nabla_{w\left(\delta_{1}\right)} w\left(\delta_{2}\right)=$ $w^{2}\left(\delta_{4}\right)+w\left(\delta_{5}\right) \in \mathscr{G}_{1}+\mathscr{G}_{2}$.

Proof of ii). For $\delta_{1}, \delta_{2}, \delta_{3}$ of $\mathscr{G}_{0}$, let us compute $\tilde{I}\left(\nabla_{\delta_{1}} w\left(\delta_{2}\right), w\left(\delta_{3}\right)\right)$. As in the case of i) one may check that $\tilde{I}\left(\nabla_{\delta_{1}} w\left(\delta_{2}\right), w\left(\delta_{3}\right)\right) \in R_{1}$.

By a similar argument to the proof of i), one may deduce that $\nabla_{\delta_{1}} w\left(\delta_{2}\right) \in \mathscr{G}_{0} \oplus \mathscr{G}_{1}$.

Proof of iii). For $\delta_{1}, \delta_{2}, \delta_{3} \in \mathscr{G}_{0}$, let us compute $\tilde{I}\left(\nabla_{w\left(\delta_{1}\right)} \delta_{2}, \delta_{3}\right) \in R_{1}$. One may show that $\nabla_{w\left(\delta_{1}\right)} \delta_{2} \in \mathscr{G}_{0} \oplus \mathscr{G}_{1}$ as in the proof of i) and ii). q.e.d.

(8.5) Let us fix an element $\zeta \in \operatorname{Der}_{R}\left(\log \Delta^{2}\right)$. Then from (8.4), we obtain $R$-homomorphisms,

$$
\begin{aligned}
\nabla_{\zeta}: & \operatorname{Der}_{R} \rightarrow \operatorname{Der}_{R}: \delta \mapsto \nabla_{\delta} \zeta \\
& \operatorname{Der}_{R}\left(\log \Delta^{2}\right) \rightarrow \operatorname{Der}_{R}\left(\log \Delta^{2}\right)
\end{aligned}
$$

Assertion. $\nabla E$ is the identity.

Proof. Suppose that $\delta$ and $\delta^{\prime}$ are homogeneous, so that

$$
[E, \delta]=(\operatorname{deg} . \delta) \delta,\left[E, \delta^{\prime}\right]=\left(\operatorname{deg} . \delta^{\prime}\right) \delta^{\prime}
$$

and

$$
E \tilde{I}\left(\delta^{\prime}, \delta\right)=\left(2+\operatorname{deg} . \delta^{\prime}+\operatorname{deg} . \delta^{\prime}\right) \tilde{I}\left(\delta^{\prime}, \delta\right)
$$

From the formula (8.2) i), we compute

$$
\begin{aligned}
2 \tilde{I}\left(\nabla_{\delta} E, \delta^{\prime}\right)= & \delta \tilde{I}\left(E, \delta^{\prime}\right)+E \tilde{I}\left(\delta^{\prime}, \delta\right)-\delta^{\prime} \tilde{I}(\delta, E) \\
& -\tilde{I}\left(\delta,\left[E, \delta^{\prime}\right]\right)+\widetilde{I}\left(E,\left[\delta^{\prime}, \delta\right]\right)+\widetilde{I}\left(\delta^{\prime},[\delta, E]\right) \\
= & 2 \widetilde{I}\left(\delta, \delta^{\prime}\right)+\widetilde{I}\left(E,\left[\delta^{\prime}, \delta\right]\right)-\delta^{\prime} \tilde{I}(\delta, E)+\delta \tilde{I}\left(E, \delta^{\prime}\right)
\end{aligned}
$$

On the other hand, since $\tilde{I}(E)=d I$ is a closed form (cf. (7.1)), $\delta \widetilde{I}\left(E, \delta^{\prime}\right)-\delta^{\prime} \tilde{I}(E, \delta)$ $=\delta \delta^{\prime} I-\delta^{\prime} \delta I=\left[\delta, \delta^{\prime}\right] I=\tilde{I}\left(E,\left[\delta, \delta^{\prime}\right]\right)$. This means $\tilde{I}\left(\nabla_{\delta} E, \delta^{\prime}\right)=\tilde{I}\left(\delta, \delta^{\prime}\right)$ for any $\delta$ and $\delta^{\prime}$. 


\section{§9. Torsion Free Connection $\nabla /$ with $\nabla / \widetilde{J}^{*}=0$}

In this paragraph, we introduce a connection $\nabla /$ and show the integrability of it.

(9.1) There exists uniquely a torsion free connection,

$$
\nabla /: \operatorname{Der}_{R} \times \operatorname{Der}_{R} \rightarrow \operatorname{Der}_{R}
$$

with the property, $\nabla / J^{*}=0$.

i.e. $\quad \delta_{1} \tilde{J}\left(\delta_{2}, \delta_{3}\right)=\tilde{J}\left(\nabla / \delta_{1} \delta_{2}, \delta_{3}\right)+\widetilde{J}\left(\delta_{2}, \nabla / \delta_{1} \delta_{3}\right)$.

Proof. Since the proof is the same as in (8.2), we omit it.

(9.2) Using the same argument as in the proof of (8.2), there is a formula,

$$
\begin{aligned}
\widetilde{J}\left(\nabla_{\delta_{1}} \delta_{2}, \delta_{3}\right)= & \delta_{1} \widetilde{J}\left(\delta_{2}, \delta_{3}\right)+\delta_{2} \widetilde{J}\left(\delta_{3}, \delta_{1}\right)-\delta_{3} \widetilde{J}\left(\delta_{1}, \delta_{2}\right) \\
& -\widetilde{J}\left(\delta_{1},\left[\delta_{2}, \delta_{3}\right]\right)+\widetilde{J}\left(\delta_{2},\left[\delta_{3}, \delta_{1}\right]\right)+\widetilde{J}\left(\delta_{3},\left[\delta_{1}, \delta_{2}\right]\right) .
\end{aligned}
$$

(9.3) From the FORMULA of (9.2) and (6.3), one may compute easily,

$$
\nabla /: \quad \mathscr{G}_{0} \oplus \cdots \oplus \mathscr{G}_{i} \times \mathscr{G}_{0} \oplus \cdots \oplus \mathscr{G}_{j} \rightarrow \mathscr{G}_{0} \oplus \cdots \oplus \mathscr{G}_{i+j} .
$$

Especially,

$$
\nabla /: \mathscr{G}_{0} \times \mathscr{G}_{0} \rightarrow \mathscr{G}_{0}
$$

(9.4) Theorem. The connection $\nabla /$ is integrable.

For the proof of (9.4), we prepare a lemma

(9.5) Lemma.

$$
\nabla_{w^{i}\left(\delta_{1}\right)} w^{j}\left(\delta_{2}\right) \equiv w^{i+j}\left(\nabla / \delta_{1} \delta_{2}\right) \quad \bmod \quad \mathscr{G}_{1} \oplus \cdots \oplus \mathscr{G}_{i+j-1}
$$

for $\delta_{1}, \delta_{2} \in \mathscr{G}_{0}$ and $i, j \geq 1$.

Proof. For $\delta_{1}, \delta_{2}$ and $\delta_{3} \in \mathscr{G}_{0}$, and $i, j \geq 1$, let us compute,

$$
\widetilde{I}\left(\nabla_{w^{1}\left(\delta_{1}\right)} w^{j}\left(\delta_{2}\right), \delta_{3}\right)=w^{i}\left(\delta_{1}\right) \widetilde{I}\left(w^{j}\left(\delta_{2}\right), \delta_{3}\right)+w^{j}\left(\delta_{2}\right) \widetilde{I}\left(\delta_{3}, w^{i}\left(\delta_{1}\right)\right)
$$




$$
\begin{aligned}
& -\delta_{3} \tilde{I}\left(w^{i}\left(\delta_{1}\right), w^{j}\left(\delta_{2}\right)\right)-\tilde{I}\left(w^{i}\left(\delta_{1}\right),\left[w^{j}\left(\delta_{2}\right), \delta_{3}\right]\right) \\
& +\tilde{I}\left(w^{j}\left(\delta_{2}\right),\left[\delta_{3}, w^{i}\left(\delta_{1}\right)\right]\right)+\widetilde{I}\left(\delta_{3},\left[\mathrm{w}^{i}\left(\delta_{1}\right), w^{j}\left(\delta_{2}\right)\right]\right) \\
& =w^{i}\left(\delta_{1}\right) \widetilde{J}\left(w^{j-1}\left(\delta_{2}\right), \delta_{3}\right)+w^{j}\left(\delta_{2}\right) \widetilde{J}\left(\delta_{3}, w^{i-1}\left(\delta_{1}\right)\right) \\
& -\delta_{3} \widetilde{J}\left(w^{i-1}\left(\delta_{1}\right), w^{j}\left(\delta_{2}\right)\right)-\widetilde{J}\left(w^{i-1}\left(\delta_{1}\right),\left[w^{j}\left(\delta_{2}\right), \delta_{3}\right]\right) \\
& +\widetilde{J}\left(w^{j-1}\left(\delta_{2}\right),\left[\delta_{3}, w^{i}\left(\delta_{1}\right)\right]+\widetilde{J}\left(\delta_{3}, w^{i+j-1}\left(\left[\delta_{1}, \delta_{2}\right]\right)+\delta^{\prime}\right)\right. \\
& \equiv P_{\ell}^{i+j-1}\left\{\begin{array}{c}
\delta_{1} \tilde{J}\left(\delta_{2}, \delta_{3}\right)+\delta_{2} \widetilde{J}\left(\delta_{3}, \delta_{1}\right)-\delta_{3} \tilde{J}\left(\delta_{1}, \delta_{2}\right) \\
-\widetilde{J}\left(\delta_{1},\left[\delta_{2}, \delta_{3}\right]\right)+\widetilde{J}\left(\delta_{2},\left[\delta_{3}, \delta_{1}\right]\right)+\widetilde{J}\left(\delta_{3},\left[\delta_{1}, \delta_{2}\right]\right)
\end{array}\right\} \\
& \bmod R_{i+j-2}\left(\text { Here } \delta^{\prime} \in \mathscr{G}_{0} \oplus \cdots \oplus \mathscr{G}_{i+j-2}\right. \text { cf. (6.4).) } \\
& \equiv P_{\ell}^{i+j-1} \widetilde{J}\left(\left(\nabla / \delta_{1} \delta_{2}, \delta_{3}\right) \bmod R_{i+j-2}\right. \\
& \equiv \widetilde{J}\left(w^{i+j-1}\left(\nabla / \delta_{1} \delta_{2}\right), \delta_{3}\right) \bmod R_{i+j-2}\left(\text { note that }\left(\nabla / \delta_{1} \delta_{2} \in \mathscr{G}_{0}\right)\right. \\
& \equiv \tilde{I}\left(w^{i+j}\left(\nabla / \delta_{1} \delta_{2}\right), \delta_{3}\right) \quad \bmod R_{i+j-2}
\end{aligned}
$$

Since $\nabla_{w^{\imath}\left(\delta_{1}\right)} w^{j}\left(\delta_{2}\right) \in \operatorname{Der}_{R}\left(\log \Delta^{2}\right)\left(\right.$ cf. (8.4)i)), $\nabla_{w^{i}\left(\delta_{1}\right)} w^{j}\left(\delta_{2}\right)-w^{i+j}\left(\nabla / \delta_{1} \delta_{2}\right)=$ $w\left(\delta_{4}\right)$ for a $\delta_{4} \in \operatorname{Der}_{R}$. Then the above computation implies,

$$
\widetilde{I}\left(w\left(\delta_{4}\right), \delta_{3}\right)=\widetilde{J}\left(\delta_{4}, \delta_{3}\right) \in R_{i+j-2} \text { for any } \delta_{3} \in \mathscr{G}_{0} .
$$

Since $\widetilde{J}$ is a non-degenerate $T$-bilinear form on $\mathscr{G}_{0}$ (cf. (5.2)), $\delta_{4} \in \mathscr{G}_{0} \otimes_{T} R_{i+j-2}=\mathscr{G}_{0} \oplus \cdots \oplus \mathscr{G}_{i+j-2}$, and $\left(\nabla_{w^{i}\left(\delta_{1}\right)} w^{j}\left(\delta_{2}\right)-w^{i+j}\left(\nabla / \delta_{1} \delta_{2}\right)=\right.$ $w\left(\delta_{4}\right) \in \mathscr{G}_{1} \oplus \cdots \oplus \mathscr{G}_{i+j-1}$.

The proof of Theorem (9.4). Put

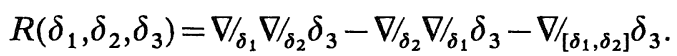

It is enough to show $R\left(\delta_{1}, \delta_{2}, \delta_{3}\right)=0$ for $\delta_{1}, \delta_{2}, \delta_{3} \in \mathscr{G}_{0}=\mathscr{G}$ (cf. (2.3) iii)). Note that $R\left(\delta_{1}, \delta_{2}, \delta_{3}\right) \in \mathscr{G}_{0}$ for $\delta_{1}, \delta_{2}, \delta_{3} \in \mathscr{G}_{0}$ (cf. (9.3) and (2.3) iii)).

$$
\begin{aligned}
& w^{3}\left(R\left(\delta_{1}, \delta_{2}, \delta_{3}\right)\right) \\
\equiv & \nabla_{w\left(\delta_{1}\right)} w^{2}\left(\nabla / \delta_{2} \delta_{3}\right)-\nabla_{w\left(\delta_{2}\right)} w^{2}\left(\nabla / \delta_{1} \delta_{3}\right) \\
& -\nabla_{w^{2}\left(\left[\delta_{1}, \delta_{2}\right]\right)} w\left(\delta_{3}\right) \quad \bmod \quad \mathscr{G}_{1} \oplus \mathscr{G}_{2} \quad \text { (cf. Lemma (9.5)) } \\
\equiv & \nabla_{w\left(\delta_{1}\right)} \nabla_{w\left(\delta_{2}\right)} w\left(\delta_{3}\right)-\nabla_{w\left(\delta_{2}\right)} \nabla_{w\left(\delta_{1}\right)} w\left(\delta_{3}\right) \\
& -\nabla_{\left[w\left(\delta_{1}\right), w\left(\delta_{2}\right)\right]} w\left(\delta_{3}\right) \quad \bmod \quad \mathscr{G}_{1} \oplus \mathscr{G}_{2}
\end{aligned}
$$




$$
=0 \text { (cf. (8.3)) }
$$

Thus $w^{3}\left(R\left(\delta_{1}, \delta_{2}, \delta_{3}\right)\right) \equiv 0 \bmod \quad \mathscr{G}_{1} \oplus \mathscr{G}_{2} \quad$ and $w^{3}\left(R\left(\delta_{1}, \delta_{2}, \delta_{3}\right)\right) \in \mathscr{G}_{3}$, and therefore $w^{3}\left(R\left(\delta_{1}, \delta_{2}, \delta_{3}\right)\right)=0$. Hence $R\left(\delta_{1}, \delta_{2}, \delta_{3}\right)=0$ for $\delta_{1}, \delta_{2}, \delta_{3} \in \mathscr{G}_{0}$.

This completes the proof of Theorem (9.4).

\section{$\S 10$. The space $\Omega=\oplus \Omega^{\left(m_{i}+1\right)}$}

In this paragraph we introduce an $\boldsymbol{R}$-vector space $\Omega$ of dimension $\ell$, with a non-degenerate inner product $J$, which we may regard as a scheme theoretic quotient variety of $V^{*}$ by the action of $W$. Our subsequent development may also be regarded as a consequence of the fact that $J$ is an everywhere flat nondegenerate quadratic form on the quotient variety $V_{\boldsymbol{C}} / W$.

(10.1) Let us fix a homogeneous generator system $P_{1}, \cdots, P_{\ell}$ of the algebra $R$ of (1) $\mathrm{v})$.

Then $\nabla / \frac{\partial}{\partial P_{i}}\left(\frac{\partial}{\partial P_{j}}\right) \in \mathscr{G}^{\left(m_{k}+1\right)}$ for $i, j=1, \cdots, \ell$, where $m_{k}$ is the smallest such that $m_{i}+m_{j}+2 \leq m_{k}+1$.

Proof. Since $\nabla / \frac{\partial}{\partial P_{i}}\left(\frac{\partial}{\partial P_{j}}\right) \in \mathscr{G}$ is homogeneous of degree $\left(m_{i}+m_{j}+2\right)$, it must be a combination of $\frac{\partial}{\partial P_{k}}$ such that $m_{i}+m_{j}+2 \leq m_{k}+1$ q.e.d.

\section{(10.2) Corollary.}

i) $\nabla /: \mathscr{G} \mathscr{G}^{\left(m_{i}+1\right)} \rightarrow \mathscr{G}^{\left(m_{i}+1\right)}$ for $i=1, \cdots, \ell$

ii) $\nabla /: \quad D^{(h)} \times \mathscr{G} \rightarrow 0, \quad \mathscr{G} \times D^{(h)} \rightarrow 0$

iii) $\nabla /: E \times \mathscr{G}^{\left(m_{i}+1\right)} \rightarrow \mathscr{G}^{\left(m_{i}+1\right)}, \mathscr{G}^{\left(m_{i}+1\right)} \times E \rightarrow \mathscr{G}^{\left(m_{i}+1\right)}$ for $i=1, \cdots, \ell$

i.e. $\nabla / E: \mathscr{G} \rightarrow \mathscr{G}$ is a filter preserving T-homomorphism.

Proof. Since $\mathscr{G}^{\left(m_{i}+1\right)}$ is spanned by $\frac{\partial}{\partial P_{j}}$ with $m_{j} \geq m_{i}$ (cf. (2.5)) and $D^{(h)}=c \frac{\delta}{\delta P_{\ell}}$ for a non-zero constant $c($ cf. (2.2)), i) and ii) are trivial. 
Let us present the Euler operator in a form $E=c P_{\ell} D^{(h)}+E^{\prime}$, for some $c \in \boldsymbol{R}^{*}$ and $E^{\prime} \in \mathscr{G}$. Then for any $\delta \in \mathscr{G}^{\left(m_{\imath}+1\right)}$,

$$
\begin{aligned}
\nabla / K_{E} \delta & =c P_{\ell} \nabla / D^{(h)} & \delta+\nabla / E^{\prime} \delta \\
& =\nabla / E^{\prime} \delta & (\because \mathrm{ii})) \\
& \in \mathscr{G}^{\left(m_{\imath}+1\right)} & (\because \mathrm{i}))
\end{aligned}
$$

and hence

$$
\nabla / \delta E=\nabla / E \delta-[E, \delta] \in \mathscr{G}^{\left(m_{\imath}+1\right)}
$$

(10.3) Lemma. As a T-module, $\mathscr{G}$ has uniquely a direct sum decomposition,

$$
\mathscr{G}=\bigoplus_{i} \overline{\mathscr{G}}^{\left(m_{i}+1\right)}
$$

such that

i) $\overline{\mathscr{G}}^{\left(m_{1}+1\right)}$ is a $T$-free homogeneous submodule of $\mathscr{G}$ of rank equal to $\sharp\{1 \leq j \leq \ell$ : $\left.m_{j}=m_{i}\right\}$ for $i=1, \cdots, \ell$.

ii) $\mathscr{G}^{\left(m_{\imath}+1\right)}=\bigoplus_{m_{j} \leq m_{i}} \overline{\mathscr{G}}^{\left(m_{j}+1\right)}$ for $i=1, \cdots, \ell$.

iii) The restriction of $\nabla / E$ on $\overline{\mathscr{G}}^{\left(m_{1}+1\right)}$ induces an endomorphism of it, so that

$$
\nabla /\left.E\right|_{\bar{G}\left(m_{i}+1\right)}=\left(m_{i}+1\right) \times \text { identity, for } i=1, \cdots, \ell \text {. }
$$

Proof. Since $\nabla / E$ is a filter preserving $T$-endomorphism of $\mathscr{G}$ (cf. (10.2) iii)), let us consider the endomorphism of $\mathrm{Gr} .\left(\mathscr{G}^{(*)}\right)$ induced by $\nabla / E$.

$$
\begin{aligned}
\nabla / \frac{\partial}{\partial P_{i}} E & =\left[\frac{\partial}{\partial P_{i}}, E\right]+\nabla / E \frac{\partial}{\partial P_{i}} \\
& =\left(m_{i}+1\right) \frac{\partial}{\partial P_{i}}+\sum_{j=1}^{\ell}\left(m_{j}+1\right) P_{j} \nabla / \frac{\partial}{\partial P_{j}} \frac{\partial}{\partial P_{i}} \\
& \equiv\left(m_{i}+1\right) \frac{\partial}{\partial P_{i}} \bmod \quad \mathscr{G}^{\left(m_{i}^{+}+1\right)} \quad \text { (cf. (10.1)). }
\end{aligned}
$$


Thus

*) $\quad \nabla / \delta E \equiv\left(m_{i}+1\right) \delta \bmod \mathscr{G}^{\left(m_{i}^{+}+1\right)}$ for any $\delta \in \mathscr{G}^{\left(m_{i}+1\right)}$.

Now let us define,

$$
\overline{\mathscr{G}}^{\left(m_{i}+1\right)}:=\left\{\delta \in \mathscr{G}: \nabla / \delta E=\left(m_{i}+1\right) \delta\right\}
$$

which is the eigenspace of the operator $\nabla / E$ for the eigenvalue $m_{i}+1$. By definition, $\overline{\mathscr{G}}^{\left(m_{i}+1\right)}$ is a homogeneous $T$-submodule of $\mathscr{G}$.

Now let us show ii) by a descending induction on $m_{i}$. Since $\mathscr{G}^{(\infty)}$ and $\overline{\mathscr{G}}^{(\infty)}$ are zero, we may assume that

$$
\mathscr{G}^{\left(m_{i}^{+}+1\right)}=\bigoplus_{m_{j} \geq m_{i}^{+}} \overline{\mathscr{G}}^{\left(m_{j}+1\right)} \quad \text { for a } 1 \leq i \leq \ell
$$

Then by this induction hypotheses and the formula $*)$, for any $\delta$ of $\mathscr{G}^{\left(m_{1}+1\right)}$, we have a presentation

$$
\nabla / \delta E=\left(m_{i}+1\right) \delta+\sum_{m_{j} \geq m_{i}^{+}} \delta_{j}, \text { where } \delta_{j} \in \overline{\mathscr{G}}^{\left(m_{j}+1\right)}
$$

Put $\quad \delta_{i}=\delta-\sum_{m_{j} \geq m_{i}^{+}} \frac{1}{m_{j}-m_{i}} \delta_{j}$. One computes easily,

$$
\begin{aligned}
\nabla / \delta_{i} E & =\nabla / \delta E-\sum_{j} \frac{1}{m_{j}-m_{i}} \nabla / \delta_{j} E \\
& =\left(m_{i}+1\right) \delta+\sum_{j} \delta_{j}-\sum_{j} \frac{m_{j}+1}{m_{j}-m_{i}} \delta_{j} \\
& =\left(m_{i}+1\right)\left\{\delta-\sum_{j} \frac{1}{m_{j}-m_{i}} \delta_{j}\right\}=\left(m_{i}+1\right) \delta_{i} .
\end{aligned}
$$

Thus $\delta=\delta_{i}+\sum_{j} \frac{1}{\mathrm{~m}_{j}-\mathrm{m}_{i}} \delta_{j} \in \underset{m_{j} \geq m_{i}}{\bigoplus} \overline{\mathscr{G}}^{\left(m_{j}+1\right)}$, and the assertion ii) is proven. Together with (2.5) ii), one shows easily the assertion i) and the lemma is proven. 
(10.4) Definition. Put,

$\Omega^{\left(m_{i}+1\right)}:=\left\{\delta \in \overline{\mathscr{G}}^{\left(m_{2}+1\right)}: \delta\right.$ is homogeneous of degree $\left.-\left(m_{i}+1\right)\right\}$

for $i=1, \cdots, \ell$ and put

$$
\Omega:=\bigoplus_{i} \Omega^{\left(m_{\imath}+1\right)} \subset \mathscr{G} \subset \operatorname{Der}_{R}
$$

\section{(10.5) Theorem.}

i) $\Omega^{\left(m_{i}+1\right)}$ is an $\boldsymbol{R}$-vector space of dimension equal to $\#\left\{1 \leq j \leq \ell ; m_{j}=m_{i}\right\}$, such that $\overline{\mathscr{G}}^{\left(m_{i}+1\right)}=\Omega^{\left(m_{1}+1\right)} \otimes_{R} T$. Hence $\Omega$ is an $R$-vector space of dimension $\ell$, such that $\mathscr{G}=\Omega \bigotimes_{\mathrm{R}} T$ and $\operatorname{Der}_{R}=\Omega \bigotimes_{\mathrm{R}} R$.

ii) $\Omega$ is integrable, in the following sense. For any $\delta_{1}$ and $\delta_{2} \in \Omega$, the bracket product $\left[\delta_{1}, \delta_{2}\right]=0$.

iii) The restriction of the T-bilinear form $\tilde{J}$ on the vector space $\Omega \times \Omega$ takes constant values in $\boldsymbol{R}$, so that we get an $\boldsymbol{R}$-bilinear form

$$
J: \Omega \times \Omega \rightarrow R .
$$

Proof. Take a projection of $\frac{\partial}{\partial P_{i}} \in \mathscr{G}^{\left(m_{i}+1\right)}$ to the factor $\overline{\mathscr{G}}^{\left(m_{i}+1\right)}$, and denote it by $D_{i}$. Since deg. $\left(\frac{\partial}{\partial P_{i}}\right)=-\left(m_{i}+1\right)$, we also have deg. $\left(D_{i}\right)=$ $-\left(m_{i}+1\right)$ and $D_{i} \in \Omega^{\left(m_{1}+1\right)}$. Because of the description of (2.5), one may easily check that $\Omega^{\left(m_{i}+1\right)}$ is spanned by $D_{j}$ with $m_{j}=m_{i}$ as an $R$-vector space and that $\overline{\mathscr{G}}^{\left(m_{i}+1\right)}=\Omega^{\left(m_{i}+1\right)} \otimes_{R} T$. Other statements of i) are direct consequences of the above statement.

Proof of ii) and iii).

Assertion 1. $\nabla / E \Omega=0$.

Proof. Take an element $\delta \in \Omega^{\left(m_{2}+1\right)}$. Then $\nabla / E \delta=\nabla / \delta E+[E, \delta]=\left(m_{i}+1\right) \delta$ $-\left(m_{i}+1\right) \delta=0$.

Assertion 2. $\Omega=\operatorname{ker}\left(\nabla / E: \operatorname{Der}_{R} \rightarrow \operatorname{Der}_{R}\right)$. 
Proof. Since $\Omega \subset \operatorname{ker}(\nabla / E)$ is shown in Assertion 1, let us show the converse inclusion.

Because of i) of (10.5); we may present any element $\delta \in \operatorname{Der}_{R}$, in the form $\sum_{i=1}^{\ell} \varphi_{i} D_{i}$, for some $\varphi_{i} \in R, i=1, \cdots, \ell$. Suppose that $\nabla / E \delta=\sum_{i}\left(E \varphi_{i}\right) D_{i}=0$ (cf. Assertion 1). Since $D_{i}$ are $R$-linearly independent, we get $E \varphi_{i}=0$, $i=1, \cdots, \ell$. This is possible only when $\varphi_{i}, i=1, \cdots, \ell$ are constants in $R$. (cf. (7.2) ii)).

Assertion 3. $\Omega=\left\{\delta \in \operatorname{Der}_{R}: \nabla / \delta^{\prime} \delta=0\right.$ for any $\left.\delta^{\prime} \in \operatorname{Der}_{R}\right\}$.

Proof. Because of Assertion 2, we have the inclusion,

$$
\Omega \supset\left\{\delta \in \operatorname{Der}_{R}: \nabla / \delta^{\prime} \delta=0 \text { for any } \delta^{\prime} \in \operatorname{Der}_{R}\right\}
$$

Because of Theorem (9.4), The right-hand of the above inclusion has $\boldsymbol{R}$-dimension $\ell$, which is equal to $\operatorname{dim}_{\mathbb{R}} \Omega$ (cf. (10.5) i)).

Assertion 4. For $\delta_{1}$ and $\delta_{2}$ of $\Omega,\left[\delta_{1}, \delta_{2}\right]=0$.

Proof. Because of the torsionfreeness of $\nabla /$ and the above Assertion 3, $\left[\delta_{1}, \delta_{2}\right]=\nabla / \delta_{1} \delta_{2}-\nabla / \delta_{2} \delta_{1}=0$.

Assertion 5. The restriction of the form $\tilde{J}$ on $\Omega \times \Omega$ takes constant values in $R$.

Proof. For $\delta, \delta^{\prime} \in \Omega$, let us compute

$$
E\left(\widetilde{J}\left(\delta, \delta^{\prime}\right)\right)=\widetilde{J}\left(\nabla / E \delta, \delta^{\prime}\right)+\widetilde{J}\left(\delta, \nabla / E \delta^{\prime}\right)=0 \quad \text { (by Assertion 1). }
$$

Then $E\left(\widetilde{J}\left(\delta, \delta^{\prime}\right)\right)=0$ is possible only when $\widetilde{J}\left(\delta, \delta^{\prime}\right)$ is a constant.

This completes the proof of the theorem (10.5).

(10.6) The uniqueness of $\Omega$.

Let $\Omega^{\prime} \subset \operatorname{Der}_{R}\left[\Delta^{-2}\right]$ be a real $\ell$-dimensional subspace such that

i) $\operatorname{Der}_{R}\left[\Delta^{-2}\right]=\Omega^{\prime} \otimes_{R} R\left[\Delta^{-2}\right]$.

ii) $\Omega^{\prime}$ is integrable. That is: $\left[\delta, \delta^{\prime}\right]=0$ for $\delta, \delta^{\prime} \in \Omega^{\prime}$.

iii) The restriction of $\tilde{J}$ on $\Omega^{\prime} \times \Omega^{\prime}$ takes constant values. 
Then $\boldsymbol{\Omega}^{\prime}=\boldsymbol{\Omega}$.

Proof. For $\delta_{1}, \delta_{2}, \delta_{3} \in \Omega^{\prime}$, using the conditions ii) and iii) let us calculate

$$
\begin{aligned}
\widetilde{J}\left(\nabla / \delta_{1} \delta_{2}, \delta_{3}\right) & =\delta_{1} \widetilde{J}\left(\delta_{2}, \delta_{3}\right)+\delta_{2} \widetilde{J}\left(\delta_{3}, \delta_{1}\right)-\delta_{3} \widetilde{J}\left(\delta_{1}, \delta_{2}\right) \\
& -\widetilde{J}\left(\delta_{1},\left[\delta_{2}, \delta_{3}\right]\right)+\widetilde{J}\left(\delta_{2},\left[\delta_{3}, \delta_{1}\right]\right)+\widetilde{J}\left(\delta_{3},\left[\delta_{1}, \delta_{2}\right]\right) \\
& =0
\end{aligned}
$$

Because of the condition i) and the non-degeneracy of $\tilde{J}$, we get $\nabla / \delta_{1} \delta_{2}=0$ for $\delta_{1}, \delta_{2} \in \Omega^{\prime}$. Again by the condition i) $\nabla / \delta_{\delta} \delta_{2}=0$ for any $\delta \in \operatorname{Der}_{R}$ and $\delta_{2} \in \Omega^{\prime}$. Comparing this with Assertion 3 of the proof of the theorem (10.5), we get $\Omega=\Omega^{\prime}$.

q.e.d.

(10.7) Now let us show that we may regard $R$ as the symmetric algebra of the $R$-dual vector space $\Omega^{*}$ of $\Omega$.

Theorem. Put

$\Omega^{*}:=\left\{P \in R^{+}(\right.$elements of positive degree $) ; \delta P \in \boldsymbol{R}$ for any $\left.\delta \in \Omega\right\}$.

Then i) $\Omega^{*}$ is an $R$-dual vector space of $\Omega$ by the pairing, $\Omega \times \Omega^{*} \ni(\delta, P) \mapsto$ $\delta P \in \boldsymbol{R}$.

ii) $R$ is generated by $\Omega^{*}$ as an $\boldsymbol{R}$-algebra

iii) Let us decompose, $\Omega^{*}=\bigoplus_{i} \Omega^{*\left(m_{1}+1\right)}$, which is dual to the decomposition of (10.4). Then any element of $\Omega^{*\left(m_{i}+1\right)}$ is homogeneous of degree $m_{i}+1$, for $i=1, \cdots, \ell$.

Proof. By using the basis $D_{i}, i=1, \cdots, \ell$ of Theorem (10.5), let us develop the Euler operator $E$ in the form $E=\sum_{i=1}^{\ell}\left(m_{i}+1\right) Q_{i} D_{i}$, where $Q_{i} \in R$, $i=1, \cdots, \ell$ are homogeneous of degree $m_{i}+1$.

Since $\left[E, D_{j}\right]=-\left(m_{j}+1\right) D_{j}, j=1, \cdots, \ell$ (cf. (7.2) iii)),

$$
\begin{aligned}
\sum_{i=1}^{\ell}\left(m_{i}+1\right)\left(D_{j} Q_{i}\right) D_{i}-\left(m_{j}+1\right) D_{j} & =\sum_{i=1}^{\ell}\left(m_{i}+1\right) Q_{i}\left[D_{i}, D_{j}\right] \\
& =0(\text { cf. }(10.5) \mathrm{ii})) .
\end{aligned}
$$


Thus we get

$$
D_{j} Q_{i}=\delta_{j, i} \quad \text { (Kronecker's symbol) for } i, j=1, \cdots, \ell \text {. }
$$

This means $Q_{i} \in \Omega^{*} i=1, \cdots, \ell$. Since $\operatorname{det}\left(D_{j} Q_{i}\right)_{i, j}=1, Q_{1}, \cdots, Q_{\ell}$ must be $\mathbb{R}$-linearly independent so that they span the vector space $\Omega^{*}$, which becomes the dual space of $\Omega$.

Since $D_{j} \equiv \frac{\partial}{\partial P_{j}} \bmod \left(\frac{\partial}{\partial P_{k}}\right.$ such that $\left.m_{k}>m_{j}\right)$, one may easily compute that det. $\left(\frac{\partial Q_{i}}{\partial P_{j}}\right)_{i, j}=\operatorname{det} .\left(D_{j} Q_{i}\right)_{i, j}=1$. Hence $Q_{1}, \cdots, Q_{\ell}$ is a homogeneous generator system of the algebra $R$. Then $D_{j}=\sum_{i=1}^{\ell}\left(D_{j} Q_{i}\right) \frac{\partial}{\partial Q_{i}}=\frac{\partial}{\partial Q_{j}}$ for $j=1, \cdots, \ell$.

Thus we get a presentation

$$
\Omega^{*\left(m_{\imath}+1\right)}=\bigoplus_{m_{j}=m_{i}} R Q_{j} \quad \text { for } i=1, \cdots, \ell
$$

This completes the proof of (10.7).

\section{§11. Concluding Remarks}

As we have mentioned in the introduction, this note was planned as a part of the study of primitive integrals in [7], since the results in this note enable us to compute the Poincare duality of the vanishing cycles of rational double points by means of a duality of certain local cohomology groups which are associated to the unfolding of the singularities as follows.

(11.1) Let us remember the constructions in this note.

We started with a group $W$ and its irreducible presentation as a reflexion group acting on a real vector space $V$. After several constructions we obtained at the end a weighted vector space $\Omega=\oplus \Omega^{\left(m_{i}+1\right)}$ and an inner product $J$ (homogeneous of degree $h+2$ ) on $\Omega$ and the discriminant polynomial $\Delta^{2}$ in the symmetric algebra $R$ of $\Omega^{*}$.

Thus we get a correspondence of objects,

$$
(W, V) \mapsto\left(\Omega, J, \Delta^{2}\right) .
$$

(11.2) Assertion. The above correspondence is reversible in the following sense 
Suppose that we forget about $W$ and $V$, but are given the information on the weighted vector space $\Omega$, the inner product $J$ and the discriminant polynomial $\Delta^{2}$. Then one may reconstruct $V$ and $W$ from $\Omega, J, \Delta^{2}$ in the following algebraic way.

i) Consider the symmetric algebra $R$ of $\Omega^{*}$ (cf. (10.7)).

Using the weights of $\Omega$, we can attach a graded ring structure to $R$, so that $\Delta^{2}$ is a (weighted) homogeneous polynomial of degree $h \cdot \ell$.

ii) Consider $\operatorname{Der}_{R}, \operatorname{Der}_{R}\left(\log \Delta^{2}\right)$ and $\Omega_{R}, \Omega_{R}\left(\log \Delta^{2}\right)$, which are graded $R$-modules (cf. (2.1)).

One may naturally embed $\Omega \subset \operatorname{Der}_{R}$. As a lowest degree element of $\Omega \subset \operatorname{Der}_{R}$, one may uniquely determine an element $D^{(h)}$ up to a constant factor (cf. (2.2)).

iii) Put, $T:=\left\{P \in R: D^{(h)} P=0\right\}$

$$
\begin{aligned}
\mathscr{F} & :=\left\{\omega \in \Omega_{R}: L_{D^{(h)}} \omega=0\right\} \\
\mathscr{G} & :=\left\{\delta \in \operatorname{Der}_{R}:\left[D^{(h)}, \delta\right]=0\right\} .
\end{aligned}
$$

Then $T$ is a graded ring of dimension $\ell-1$ and $\mathscr{F}, \mathscr{G}$ are free $T$-modules of rank $\ell$. Naturally $\mathscr{F}$ and $\mathscr{G}$ are dual $T$-modules of each other.

iv) Since $\mathscr{G}=\Omega \otimes T$, we may extend $J$ to $\mathscr{G}$ as a $T$-bilinear form,

$$
\tilde{J}: \mathscr{G} \times \mathscr{G} \rightarrow T .
$$

v) As a $T$-module, we have a direct sum decomposition (cf. (6.1))

$$
\operatorname{Der}_{R}=\mathscr{G} \oplus \operatorname{Der}_{R}\left(\log \Delta^{2}\right)
$$

Then, one may define a $T$-linear map,

$$
w: \mathscr{G} \rightarrow \operatorname{Der}_{R}\left(\log \Delta^{2}\right)
$$

by $w(\delta):=$ the second factor of $P_{\ell} \delta$ in the above direct sum decomposition, where $P_{\ell}$ is any polynomial of $R$ such that $D^{(h)} P_{\ell}=1$ (cf. (6.4) i)).

vi) Let $\delta_{1}, \cdots, \delta_{\ell}$ be a $T$-free basis of $\mathscr{G}$ and let $\delta^{* 1}, \cdots, \delta^{* \ell}$ be the dual basis of $\mathscr{G}$ with respect to the inner product $\tilde{J}$.

Put,

$$
\tilde{I}^{*}:=\sum_{i=1}^{\ell} \delta_{i} \otimes w\left(\delta^{* i}\right): \Omega_{R} \times \Omega_{R} \rightarrow R
$$


Then $\tilde{I}^{*}$ is a symmetric $R$-bilinear form on $\Omega_{R}$, which does not depend onthe choice of the basis $\delta_{1}, \cdots, \delta_{\ell}$. (cf. (6.5)).

vii) Consider the Levi-Civita connection,

$$
\nabla: \operatorname{Der}_{R}\left(\log \Delta^{2}\right) \times \operatorname{Der}_{R}\left(\log \Delta^{2}\right) \rightarrow \operatorname{Der}_{R}\left(\log \Delta^{2}\right)
$$

for the metric $\tilde{I}^{*}$ (cf. (8.2), (8.3)).

Let $K$ be the algebraic closure of the quotient field of the ring $R$ so that $K \supset C$. One may naturally extend $\nabla$ to

$$
\nabla: \quad \operatorname{Der}_{R} \times\left(\operatorname{Der}_{R} \otimes_{R} K\right) \rightarrow\left(\operatorname{Der}_{R} \otimes_{R} K\right)
$$

viii) Put

$$
\begin{aligned}
& V^{*}:=\left\{\delta \in \operatorname{Der}_{R} \otimes_{R} K: \nabla_{\delta^{\prime}} \delta=0 \text { for any } \delta^{\prime} \in \operatorname{Der}_{R}\right\} \\
& V:=\left\{P \in K: \delta P \in C \text { for any } \delta \in V^{*} \text { and homogeneous of deg. } P>0\right\} .
\end{aligned}
$$

Then $V$ and $V^{*}$ are dual complex vector spaces of dimension $\ell$, consisting of elements of degree 1 and -1 respectively.

ix) Consider the symmetric algebra $S \subset K$ generated by $V$. Then $R \subset S$.

$\mathrm{x}$ ) The extension of the quotient field of $R$ by the quotient field of $S$ is a Galois extension. Let us denote by $W$ the Galois group of the extension.

The operation of the group $W$ preserves the vector space $V$ so that we obtain a pair $(W, V)$ of a group $W$ and its presentation in $G L(V)$. This is the one we are seeking.

(11.3) Note that by the above correspondence, $\left(\Omega, J, \Delta^{2}\right) \mapsto(W, V)$, the information of $\left(\Omega, J, \Delta^{2}\right)$ does not contain explicit information on the group $W$.

Roughly speaking the information of $W$ comes through the monodromy representation of the fundamental group of the complement of the discriminant locus $\Delta^{2}=0$ in the complexification of $\Omega$.

If one may go through complex analytic geometry, the group $W$ can be rather easily constructed as follows (cf. [3], [4]).

Let $\Omega^{c}$ be the complexification of the real vector space $\Omega$ and let $D$ be the hypersurface of $\Omega^{c}$ defined by the equation $\Delta^{2}=0$.

Let $g$ be a homotopy class of a path in $\Omega^{c}-D$, which turns once around a general (smooth) point of $D$ and let $N$ be the smallest normal subgroup 
of $\pi_{1}\left(\Omega^{c}-D,{ }^{*}\right)$ containing $g^{2}$.

Then $W$ is isomorphic to $\pi_{1}\left(\Omega^{c}-D,{ }^{*}\right) / \mathrm{N}$. As a complex variety, the complexification $E$ of the real vector space $V^{*}$ is characterized by the following properties,

i) $E$ is a normal irreducible analytic space.

ii) There exists a proper finite map $\pi: E \rightarrow \Omega^{c}$ such that $\left.\pi\right|_{E-\pi^{-1}(D)}$ : $E-\pi^{-1}(D) \rightarrow \Omega^{c}-D$ is an unramified covering map corresponding to the normal subgroup $N$.

As a covering transformation group, $W$ operates naturally on $E-\pi^{-1}(D)$. The uniqueness of $E$ of the properties i) and ii) guarantee that the operation of $W$ on $E-\pi^{-1}(D)$ can be extended to the operation on $E$, so that we get a natural inclusion $W \subset \operatorname{Aut}(E)$.

(11.4) Now let us compute the intersection form $I^{*}$ of vanishing cycles of simple singularities as an application of our theory. For detailed explanations and proofs of iv), v), vi) below, one is referred to [7] and [42].

i) Let $g$ be a complex simple Lie algebra and let $G$ be the adjoint group of $\mathfrak{g}$. Let $\mathfrak{h}$ be a Cartan subalgebra of $\mathfrak{g}$. The adjoint action of $G$ on $\mathfrak{g}$ induces a Weyl group $W$ action on $\mathfrak{h}$. It is a well known theorem of Chevalley that the restriction of a polynomial function on $\mathfrak{g}$ to the subspace $\mathfrak{h}$ induces an isomorphism of the invariant rings,

$$
C\left[\mathfrak{g}^{*}\right]^{G} \simeq C\left[\mathfrak{h}^{*}\right]^{W}
$$

(Here we denote by $C\left[E^{*}\right]$ the ring of polynomial functions on a vector space $E$.)

Since $W$ is a finite reflection group acting on a real form $\mathfrak{h}_{\boldsymbol{R}}$ of the algebra $\mathfrak{h}$, we may apply Theorems (10.5), (10.6) and (10.7) for $\left(\mathfrak{h}_{\boldsymbol{R}}, W\right)$. We shall use the same notations $R, \operatorname{Der}_{R}, D^{(h)}, \Delta^{2}, \mathfrak{h}, \tilde{J}, \operatorname{Der}_{R}\left(\log \Delta^{2}\right), \Omega, \cdots$, etc. (recall §2-5 and 10) for this complexified situation. So $R$ denotes the invariant ring $C\left[\mathfrak{h}^{*}\right]^{W}$ and $\operatorname{Der}_{R}$ is the module of its derivations. Then $\Omega$ is the unique complex vector space of dimension $\ell:=\operatorname{dim}_{\boldsymbol{C}} \mathfrak{h}$ characterized by the next properties a)-c):

a) there exists an inclusion of the dual vector space $\Omega^{*}$ into $C\left[\mathfrak{b}^{*}\right]^{W}$, so that one has an isomorphism:

$$
C\left[\Omega^{*}\right] \simeq C\left[\mathfrak{h}^{*}\right]^{W},
$$


b) there exists a direct sum decomposition, $\Omega=\oplus \Omega^{\left(m_{i}+1\right)}$, so that any element of the factor $\Omega^{*\left(m_{t}+1\right)}$ is a homogeneous function of degree $m_{i}+1$ on $\mathfrak{h}$ (and hence on $\mathfrak{g}$ ).

c) there exists a non-degenerate bilinear form,

$$
J: \Omega \times \Omega \rightarrow \mathbb{C}
$$

which is related to the Cartan-Killing form $I$, by the formulas of (6.5) and (5.2).

d) From a) and b) together, we obtain a quasi-homogeneous mapping,

$$
\mathfrak{g} \rightarrow \Omega\left(\xi \in \mathfrak{g} \mapsto\left(P \in \Omega^{*} \mapsto P(\xi) \in C\right) \in \Omega^{* *}\right),
$$

which we shall call an invariant morphism.

ii) Brieskorn [2] and Slodowy [10] gave a description of the universal deformation $X \rightarrow S$ of simple singularities of types $A_{\ell}, D_{\ell}$ and $E_{\ell}$ in terms of corresponding simple Lie algebra, where the total space $X$ is an affine linear subspace of $g$ of dimension $\ell+2$ transversal to the nilpotent variety, the base space $S$ is the quotient space $\mathfrak{h} / W$ which will be identified with $\Omega$ by i) a), and the deformation map is given by the restriction of the invariant map i) d). The map $X \rightarrow S$ is quasi-homogeneous of type $\left(m_{1}+1, \cdots, m_{\ell}+1 ; m_{1}+1, \cdots, m_{\ell-1}+1,1,1,1\right)$ for $r=\ell+2$ (cf. Varadarajan [12], Kostant [6]).

iii) The composition map $X \rightarrow S \simeq \Omega \rightarrow \Omega / C D^{(h)}$ is easily seen to be submersive so that the inverse image $X_{0}$ of $0 \in \Omega / C D^{(h)}$ is isomorphic to a three dimensional affine space. The restriction of the invariant map to $X_{0} \rightarrow C$ is denoted by $f$. Let $P_{1}, \cdots, P_{\ell}$ be a system of linear coordinates of $\Omega$ such that $\operatorname{deg}\left(P_{i}\right)=m_{i}+1$. Their pull backs on $X$ by the invariant map are denoted by the same $P_{1}, \cdots, P_{\ell}$. We can choose three homogeneous polynomials $X, Y$ and $Z$ of degree 1 on $X$ such that $d X \wedge d Y \wedge d Z \wedge d P_{1}$ $\wedge \cdots \wedge d P_{\ell-1} \neq 0$ every where on $X$. The map $\left(X, Y, Z, P_{1}, \cdots, P_{\ell}\right)$ defines an embedding of $X$ into $\mathbb{C}^{3} \times \Omega$, whose image is a hypersurface defined by a single equation of the form:

$$
P_{\ell}-F\left(X, Y, Z, P_{1}, \cdots, P_{\ell-1}\right)=0 .
$$

This is the universal unfolding of $f(X, Y, Z)=F(X, Y, Z, 0, \cdots, 0)$, where $f(X, Y, Z)=0$ defines the simple singularity of the corresponding type.

iv) a) Let us now compute the middle cohomology groups of the fibers 
of the mapping $X \rightarrow \Omega$, in the following two different ways and let us study the difference of them.

$$
\begin{aligned}
\mathscr{H}_{F}^{(1)} & :=\Omega_{X}^{l+2} / d P_{1} \wedge \cdots \wedge d P_{\ell} \wedge d \Omega_{X}^{1} \\
\mathscr{H}_{F}^{(0)} & :=\Omega_{X}^{2} / d \Omega_{X}^{1}+\sum_{i=1}^{\ell} d P_{i} \wedge \Omega_{X}^{1} \\
\Omega_{F} & :=\Omega_{X}^{\ell+2} / d P_{1} \wedge \cdots \wedge d P_{\ell} \wedge \Omega_{X}^{2}
\end{aligned}
$$

(Here $\Omega_{X}^{p}$ is the $C[X]$ module of polynomial $p$-forms on $X$.)

Then $\mathscr{H}_{F}^{(0)}$ and $\mathscr{H}_{F}^{(1)}$ are $R$-free modules of rank $\ell$ and $\Omega_{F}$ is an $R$-torsion module and is a $T$-free module of rank $\ell$.

We have a natural $R$-exact sequence,

$$
0 \rightarrow \mathscr{H}_{F}^{(0)} \stackrel{d P_{1} \wedge \cdots \wedge d P_{\ell}}{\longrightarrow} \quad \mathscr{H}_{F}^{(1)} \rightarrow \Omega_{F} \rightarrow 0
$$

b) Parallel to the above situation one may compute the cohomology groups of the fibers of the one parameter family $f: X_{0} \rightarrow C$, as follows

$$
\begin{aligned}
\mathscr{H}_{f}^{(1)} & :=\Omega_{X_{0}}^{3} / d f \wedge d \Omega_{X_{0}}^{1} \\
\mathscr{H}_{f}^{(0)} & :=\Omega_{X_{0}}^{2} / d \Omega_{X_{0}}^{1}+d f \wedge \Omega_{X_{0}}^{1} . \\
\Omega_{f} & :=\Omega_{X_{0}}^{3} / d f \wedge \Omega_{X_{0}}^{2}
\end{aligned}
$$

Then $\mathscr{H}_{f}^{(1)}$ and $\mathscr{H}_{f}^{(0)}$ are $C\left[P_{\ell}\right] \simeq R /\left(P_{1}, \cdots, P_{\ell-1}\right) R$-free modules of rank $\ell$ and $\Omega_{f}$ is a $C$-vectorspace of rank $\ell$.

We have the following natural identifications of two exact sequences

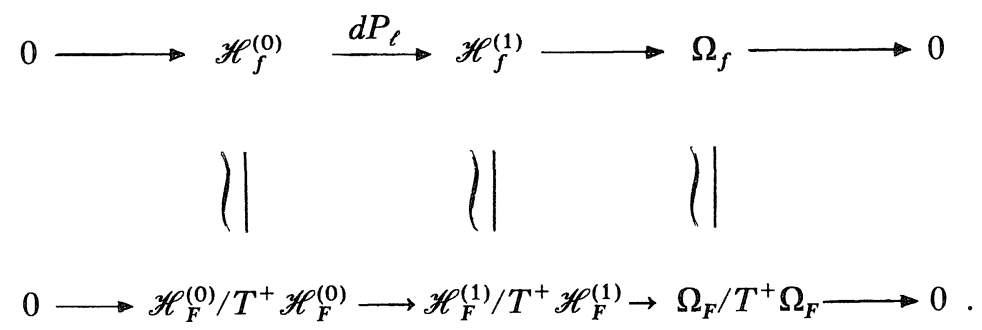

Here $T^{+}=\left(P_{1}, \cdots, P_{\ell-1}\right) T$ is the maximal ideal consisting of all positive degree 
elements of $T$.

v) a) Let us define a non-degenerate $T$-bilinear form, $\langle\rangle:, \Omega_{F} \times \Omega_{F} \rightarrow$ $T$.

Let $\omega_{i} \in \Omega_{F}, i=1,2$, be represented by forms $\varphi_{i} d X d Y d Z d P_{1} \cdots d P_{\ell-1} \in$ $\Omega_{X}^{\ell+2}, i=1,2$. Then

$$
\left\langle\omega_{1}, \omega_{2}\right\rangle:=\operatorname{Res}_{X / \Omega_{1}}\left[\begin{array}{c}
\varphi_{1} \varphi_{2} d X d Y d Z \\
\frac{\partial F}{\partial X}, \frac{\partial F}{\partial Y}, \frac{\partial F}{\partial Z}
\end{array}\right]
$$

This definition depends neither on the presentations of $\omega_{i}$ nor on the choice of the coordinates $X, Y, Z$.

b) We define also a non-degenerate $C$-bilinear form, $\langle\rangle:, \Omega_{f} \times \Omega_{f} \rightarrow C$.

Let $\omega_{i} \in \Omega_{f}, i=1,2$, be represented by forms $\varphi_{i} d X d Y d Z \in \Omega_{X_{0}}^{3}, i=1,2$. Then

$$
\left\langle\omega_{1}, \omega_{2}\right\rangle:=\operatorname{Res}_{X_{0} / 0}\left[\begin{array}{c}
\varphi_{1} \varphi_{2} d X d Y d Z \\
\frac{\partial f}{\partial X}, \frac{\partial f}{\partial Y}, \frac{\partial f}{\partial Z}
\end{array}\right]
$$

Evidently by definition, we have a commutative diagram,

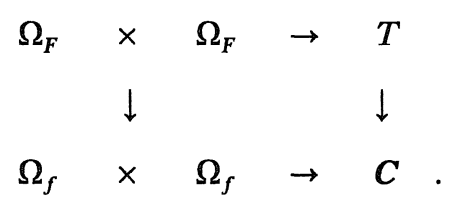

vi) Let us denote by $\nabla^{\prime}$ the Gauss-Manin connection of the family $X \rightarrow \Omega$, which is a covariant derivative on the module $\mathscr{H}_{F}^{(0)}\left[\Delta^{-2}\right]=\mathscr{H}_{F}^{(1)}\left[\Delta^{-2}\right]$. We know that $\nabla^{\prime}$ has logarithmic poles in a suitable sense.

Let $\zeta^{(0)} \in \mathscr{H}_{F}^{(0)}$ be a class presented by a form

$$
a X d Y d Z+b Y d Z d X+c Z d X d Y, \text { where } a, b, c
$$

are constants such that $a+b+c=1$.

Theorem. The covariant derivation $\theta^{(0)}:=\nabla^{\prime} \zeta^{(0)}$ of $\zeta^{(0)}$ induces an $R$-isomorphism of exact sequences, 


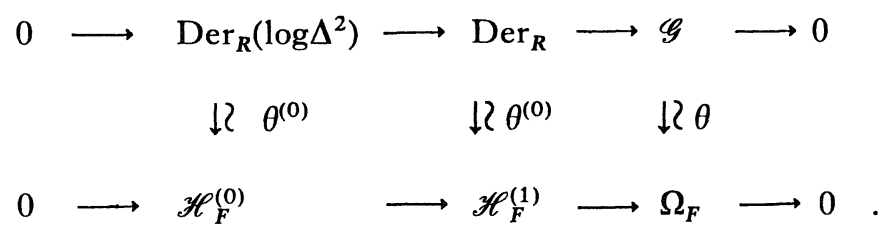

The above isomorphism $\theta: \mathscr{G} \simeq \Omega_{F}$ induces an identification of $T$-bilinear forms $\tilde{J}$ of (5.2), (6.5) and $\langle$,$\rangle of v) a) of (11.4).$

Corollary. The above isomorphism $\mathscr{G} \simeq \Omega_{F}$ induces an isomorphism of vector spaces $\Omega \simeq \Omega_{f}$. By this isomorphism, the bilinear form $J$ on $\Omega$ is identified with the bilinear form $\langle$,$\rangle of \mathrm{v}) \mathrm{b}$ ) of (11.4).

Now let $\zeta^{(1)}=\nabla_{D^{(h)}}^{\prime(0)} \in \mathscr{H}_{F}^{(1)}$ be the class represented by the form $d X d Y d Z d P_{1} \cdots d P_{\ell-1} \in \Omega_{X}^{\ell+2}$

Theorem. The covariant derivation $\theta^{(1)}:=\nabla^{\prime} \zeta^{(1)}$ of $\zeta^{(1)}$ induces an $R$-isomorphism of exact sequences

$$
\begin{aligned}
& 0 \longrightarrow \operatorname{Der}_{R}\left(\log \Delta^{2}\right) \longrightarrow \operatorname{Der}_{R} \longrightarrow \mathscr{G} \longrightarrow 0 \\
& 12 \theta^{(1)} \quad 12 \theta^{(1)} \quad 12 \theta \\
& 0 \longrightarrow \mathscr{H}_{F}^{(1)} \quad \longrightarrow \mathscr{H}_{F}^{(2)} \longrightarrow \Omega_{F} \longrightarrow 0
\end{aligned}
$$

(Here $\mathscr{H}_{F}^{(2)}:=\nabla_{D^{(h)}}^{\prime} \mathscr{H}_{F}^{(1)} \subset \mathscr{H}_{F}^{(1)}\left[\Delta^{-2}\right]$ is an $R$-free module of rank $\ell$.)

The above isomorphism $\theta^{(1)}$ induces an identification of the connection $\nabla$ of (8.2) with the Gauss-Manin connection $\nabla^{\prime}$.

Corollary. Let $\delta^{1}, \cdots, \delta^{\ell}$ and $\delta^{1 *}, \cdots, \delta^{\ell *}$ be dual T-free bases of $\mathscr{G}$ with respect to the form $\tilde{J}$, and let us put $I^{*}=\sum_{i=1}^{\ell} \delta^{i} \otimes w\left(\delta^{i *}\right)$.

Then $\theta^{(1)}\left(I^{*}\right) \in \mathscr{H}_{F}^{(1)} \bigotimes_{R} \mathscr{H}_{F}^{(2)}$ is the intersection form on the middle homology group of the regular fibers of the mapping $X \rightarrow \Omega$.

Note. Combining the above corollaries with (11.2), one sees that only the information of the vector space $\Omega_{f}=\Omega_{X_{0}}^{3} / d f \wedge \Omega_{X_{0}}^{2}$ with its bilinear form $\langle\rangle:, \Omega_{f} \times \Omega_{f} \rightarrow C$ of $\left.\mathrm{v}\right)$ b) and the information of the discriminant $\Delta^{2} \in C\left[\Omega_{f}^{*}\right]$ of the mapping $X \rightarrow \Omega_{f}$ together suffice for the calculation of the 
intersection form $I$ (or $I^{*}$ ). Then the vector space $V$ may be regarded as the middle cohomology group of a generic fiber of the map $X \rightarrow \Omega$.

\section{References}

[1] Bourbaki, N., Groupes et algèbres de Lie Chap. 4, 5 et 6, Hermann, Paris, 1968.

[2] Brieskorn, E., Singular elements of semi-simple algebraic groups, Actes Congres Intern. Math., 2 (1970), 279-284.

[ 3 ] — Die Fundamentalgruppe des Raumes der regularen, Orbits einer enddlichen komplexen Spiegelungsgruppe, Invent. Math., 12 (1971), 57-61.

[ 4 ] Brieskorn, E. and Saito, K., Artin-Gruppen und Coxeter-Gruppen, Invent. Math., 17 (1972), 245-271.

[ 5 ] Chevalley, C., Invariants of finite groups generated by reflexions, Amer. J. Math., 77 (1955), 778-782.

[6] Kostant, B., The principal three-dimensional subgroup and the Betti numbers of a complex simple group, Amer. J. Math., 81 (1959), 973-1032.

[ 7 ] Saito, K., Period Mapping Associated to a Primitive Form, Publ. RIMS, Kyoto Univ., 19 (1983), 1231-1264

[8] Theory of logarithmic differential forms and logarithmic vector fields, J. Fac. Sci., Univ. Tokyo Sci. IA, 27 (1980), 265-291.

[9] Saito, K., Yano, R. and Sekiguchi, J., On a certain generator system of the ring of invariants of a finite reflexion group, Comm. Algebra, 8 (1980), 373-408.

[10] Slodowy, P., Einfache Singularitäten und einfache algebraische Gruppen, Regensburger Math. Schriften, 2 (1978).

[11] Steinberg, R., Invariants of finite reflexion groups, Canad. J. Math., 12 (1960), 616-618.

[12] Varadarajan, V.S., On the ring of invariant polynomials on a semisimple Lie algebra, Amer. J. Math., 90 (1968), 308-317.

[13] Anbai, T., Explicit solutions of K. Saito's system of differential quations (the case of simple singularities of types A and D), Master's thesis, Tohoku Univ., March, 1987. This article is in the appendix of Tadao Oda: Introduction to algebraic singularities, Lect. Note. at Inst. of Math. Sci., Madras, 1987-1988, unpublished.

[14] Blok, B. and Varchenko, A., Topological conformal field theories and the flat coordinates, Preprint IASSNS-HEP-91/5, Jan. 1991.

[15] Coleman, A.J., The Betti numbers of the simple groups, Canad. J. Math., 10 (1958), 349-356.

[16] Dijkgraaf, R, Verlinde, H. and Verlinde, E., Topological Strings in $d<1$, Nuclear Phys., $\mathbb{B 3 5 2}$ (1991), 59-86.

[17] - Notes on Topological Strings Theory and 2D Quantum Gravity, PUPT-1217, IASSNS-HEP-90/80, Nov. 1990.

[18] Ishiura, S. and Noumi, M., A Calculus of the Gauss-Manin System of Type $A_{l}$. I,II, Proc. Japan Acad., 58, Ser. A (1982), 13-16, 62-65.

[19] Kato, M. and Watanabe, S., The flat coordinate system of the rational double point of $E_{8}$ type, Bull. Coll. Sci., Univ. Ryukyus. 32 (1981), 1-3.

[20] Kato M., The flat coordinates of universal unfoldings of $\tilde{E}_{6}$ and $\tilde{E}_{7}$, Bull. Coll. Sci., Univ. Ryukyus, 42 (1986) 5-10.

[21] Namikawa, Y., Higher residues associated with an isolated hypersurface singularity, Adv. Stud. Pure Math., 1 (1982), 181-193.

[22] Noumi, M., Expansions of the solution of a Gauss-Manin system at a point of infinity, Tokyo J. Math. 7, (1984), 1-60.

[23] - Flat coordinate system of type $E, \tilde{E}_{6}, \tilde{E}_{7}, \tilde{E}_{8}$, Tables (1986), unpublished. 
[24] Oda, T., K. Saito's period map for holomorphic functions with isolated critical points, Adv. Stud. Pure Math. 10, 1987, Algebraic Geometry, Sendai, (1985), 591-648.

[25] Orlik, P., Discriminants in the invariant theory of reflection groups, Nagoya Math. J., 109 (1988), 23-45.

[26] Saito, K., Einfach-Elliptisch Singularitäten, Inventiones Math. 23, (1974), 289-325.

[27] —, On the periods of primitive integrals, Harvard (1979) unpublishhed.

[28] — The higher residue pairings $K_{F}^{(k)}$ for a family of hypersurface singular points, in Singularities (P. Orlik, ed.), Proc. Symp. in Pure Math., AMS 40, part 2, (1983), 441-463.

[29] —- Extended affine root systems I (Coxeter transformations), Publ. RIMS, Kyoto Univ., 21 (1985), 75-179, Extended affine root systems II (Flat Invariants), ibid. 26 (1990), 15-78.

[30] Saito, K. and Satake, I., On the extended affine root systems, RIMS Kōkyuroku (1991)

[31] Satake, I., Automorphisms of the extended affine root system and modular property for flat theta invariants, to appear.

[32] — Flat structure for the simply elliptic singularities and Jacobi form, to appear in the Proc. Trieste, 1991.

[33] Slodowy, P., Simple singularities and simple algebraic groups, Lect. Note. in Math., 815, Springer, 1980.

[34] Springer, A.T., Regular elements of finite reflection groups, Invent, Math., 25 (1974), 159-198.

[35] Wirthmüller, K., Root systems and Jacobi forms, Compositio Math., 82 (1992), 293-354.

[36] Yamada, H., Symplectic reduction and simultaneous resolution of simple singularities, submitted.

[37] Yano, T., Flat coordinate system for the deformation of type $E_{6}$, Proc. Japan Acad., 57, Ser A, No. 8 (1981), 412-414.

[38] - Flat coordinate system for the deformation of type $E_{7}$, Preprint 1981.

[39] — - On the invariant ring of the Weyl group of type $E_{7}$, Preprint 1981.

[40] - Free deformation for isolated singularity, Sci. Rep. Saitama Univ., Ser. A, 9 (1980), 61-70.

[41] Yano, T. and Sekiguchi, J., The microlocal structure of weighted homogeneous polynomials associated with Coxeter systems, I, II, Tokyo J. Math., 2 (1979), 193-219; 4 (1981), 1-34.

[42] Saito, K., On the identification of intersection form on the middle homology group with the flat function via period mapping, Proc. Japan Acad., 58, Ser A (1982), 196-199. 
